\title{
A POSTERIORI ERROR ANALYSIS OF THE TIME DEPENDENT NAVIER-STOKES EQUATIONS WITH MIXED BOUNDARY CONDITIONS
}

\author{
CHRISTINE BERNARDI ${ }^{\dagger}$ AND TONI SAYAH ${ }^{\ddagger}$
}

\begin{abstract}
In this paper we study the time dependent Navier-Stokes problem with mixed boundary conditions. The problem is discretized by the backward Euler's scheme in time and finite elements in space. We establish optimal a posteriori error estimates with two types of computable error indicators, the first one being linked to the time discretization and the second one to the space discretization. We finish with numerical validation experiments.

KEYWORDS. Navier-Stokes equations, mixed boundary conditions, finite element method, a posteriori analysis.
\end{abstract}

\section{InTRODUCTION.}

Let $\Omega$ be a bounded simply-connected open domain in $\mathbb{R}^{d}, d=2,3$, with a Lipschitz-continuous connected boundary $\partial \Omega$, and let $[0, T]$ denote an interval in $\mathbb{R}$ where $T$ is a positive constant. We consider a partition without overlap of $\partial \Omega$ into two connected parts $\Gamma_{m}$ and $\Gamma$, where the index "m" means membrane. Let also $\mathbf{n}$ be the unit outward normal vector to $\Omega$ on its boundary $\partial \Omega$. We intend to work with the following time dependent Stokes system:

$$
\begin{array}{ll}
\frac{\partial \mathbf{u}}{\partial t}(t, \mathbf{x})-\nu \Delta \mathbf{u}(t, \mathbf{x})+(\mathbf{u}(t, x) \cdot \nabla) \mathbf{u}(t, x)+\nabla P(t, \mathbf{x})=\mathbf{f}(t, \mathbf{x}) & \text { in }] 0, T[\times \Omega, \\
\operatorname{div} \mathbf{u}(t, \mathbf{x})=0 & \text { in }[0, T] \times \Omega, \\
\mathbf{u}(t, \mathbf{x})=\mathbf{u}_{D} & \text { on }[0, T] \times \Gamma, \\
\mathbf{u}(t, \mathbf{x}) \cdot \mathbf{n}(\mathbf{x})=u_{m} & \text { on }[0, T] \times \Gamma_{m}, \\
\operatorname{curl} \mathbf{u}(t, \mathbf{x}) \times \mathbf{n}(\mathbf{x})=\mathbf{0} & \text { on }[0, T] \times \Gamma_{m}, \\
\mathbf{u}(0, \mathbf{x})=\mathbf{u}_{0} & \text { in } \Omega,
\end{array}
$$

where $\mathbf{f}$ represents a density of body forces and the viscosity $\nu$ is a positive constant. The unknowns are the velocity $\mathbf{u}$ and the pressure $P$ of the fluid.

Indeed, the system of partial differential equations in (1.1) is provided with mixed boundary conditions which are standard Dirichlet conditions on the velocity on $\Gamma$ and conditions on the normal component of the velocity and the tangential components of the vorticity curl $\mathbf{u}$ on $\Gamma_{m}$ (note that, in dimension $d=2$, the vorticity is a scalar function which is zero on $\Gamma_{m}$ ). This kind of mixed boundary conditions are also considered in [1] and [11] for the studies of the a posteriori errors for the Stokes problem, and also in [4] for the stationary case.

The aim of this work is to extend the a posteriori estimates to the more realistic case of mixed boundary conditions and to show numerical experiments. We propose a very standard low cost discretization relying on the Euler's implicit scheme in time combined with finite elements in space, and prove optimal a posteriori error estimates for the discrete problem. To do this, we have rather follow the approach of [8] and [6] introduced in [3] which consists in uncoupling as much as possible the time and space errors in view of a simple adaptivity strategy.

April 11, 2013.

$\dagger$ Laboratoire Jacques-Louis Lions, C.N.R.S. \& Université Pierre et Marie Curie, B.C. 187, 4 place Jussieu, 75252 Paris Cedex 05, France. e-mail: bernardi@ann.jussieu.fr.

$¥$ Faculté des Sciences, Université Saint-Joseph, B.P 11-514 Riad El Solh, Beyrouth 1107 2050, Liban. e-mail: toni.sayah@fs.usj.edu.lb. 
The outline of the paper is as follows:

- Section 2 is devoted to the study of the continuous problem.

- In section 3, we introduce the discrete problem and we recall its main properties.

- In section 4 , we study the a posteriori errors and derive optimal estimates.

- In section 5 , we show numerical tests of validation.

To simplify the notation, we present all the analysis in Sections 2,3 and 4 in dimension $d=3$ (indeed, it is simpler in dimension $d=2$ ). In contrast, the numerical experiments in Section 5 are performed in dimension $d=2$.

\section{AnAlysis OF THE MODEL}

We suppose that $\partial \Gamma_{m}=\partial \Gamma$ is a Lipschitz-continuous submanifold of $\partial \Omega$. For simplicity, we work with zero boundary conditions $\mathbf{u}_{D}=\mathbf{0}, u_{m}=0$, in order to avoid the too technical proofs linked to the Hopf lemma, see [13, chap. IV, Theorem 2.3] and also zero initial conditions $\mathbf{u}_{0}=\mathbf{0}$, since the extension to the general case is rather obvious. In view of the variational formulation of Problem (1.1), we recall the formulas

$$
-\Delta \mathbf{u}=\operatorname{curl}(\operatorname{curl} \mathbf{u})-\nabla(\operatorname{div} \mathbf{u})
$$

and

$$
(\mathbf{u} \nabla \mathbf{u}) \mathbf{u}=(\operatorname{curl} \mathbf{u}) \times \mathbf{u}+\frac{1}{2} \operatorname{grad}|\mathbf{u}|^{2} .
$$

Then Problem (1.1) can equivalently be written as (we suppress the variables $\mathbf{x}$ and $t$ for brevity)

$$
\begin{array}{ll}
\frac{\partial \mathbf{u}}{\partial t}+\nu \mathbf{c u r l}(\operatorname{curl} \mathbf{u})+(\operatorname{curl} \mathbf{u}) \times \mathbf{u}+\nabla p=\mathbf{f} & \text { in }] 0, T[\times \Omega, \\
\operatorname{div} \mathbf{u}=0 & \text { in }[0, T] \times \Omega, \\
\mathbf{u} \times \mathbf{n}=\mathbf{0} & \text { on }[0, T] \times \Gamma, \\
\mathbf{u} \cdot \mathbf{n}=0 & \text { on }[0, T] \times \partial \Omega, \\
\mathbf{c u r l} \mathbf{u} \times \mathbf{n}=\mathbf{0} & \text { on }[0, T] \times \Gamma_{m} \\
\mathbf{u}=\mathbf{0} & \text { in }\{0\} \times \Omega,
\end{array}
$$

where the new unknown

$$
p=P+\frac{1}{2}|\mathbf{u}|^{2}
$$

represents the dynamic pressure. The reason for choosing this modified form is that the last boundary condition, namely curl $\mathbf{u} \times \mathbf{n}=\mathbf{0}$ on $\Gamma_{m}$, can now be treated as a natural boundary condition.

In order to write the variational formulation of the previous problem, we introduce the Sobolev spaces:

$$
W^{m, p}(\Omega)=\left\{v \in L^{p}(\Omega), \partial^{\alpha} v \in L^{p}(\Omega), \quad \forall|\alpha| \leq m\right\}, \quad H^{m}(\Omega)=W^{m, 2}(\Omega),
$$

equipped with the following semi-norm and norm :

$$
|v|_{m, p, \Omega}=\left\{\sum_{|\alpha|=m} \int_{\Omega}\left|\partial^{\alpha} v(\mathbf{x})\right|^{p} d \mathbf{x}\right\}^{1 / p} \quad \text { and } \quad\|v\|_{m, p, \Omega}=\left\{\sum_{k \leq m}|v|_{k, p, \Omega}^{p}\right\}^{1 / p} .
$$

As usual, we shall omit $p$ when $p=2$ and denote by $(\cdot, \cdot)$ the scalar product of $L^{2}(\Omega)$. We also consider the spaces

$$
H(\operatorname{div}, \Omega)=\left\{\mathbf{v} \in L^{2}(\Omega)^{3}, \operatorname{div} \mathbf{v} \in L^{2}(\Omega)\right\}
$$

and

$$
H(\operatorname{curl}, \Omega)=\left\{\mathbf{v} \in L^{2}(\Omega)^{3}, \operatorname{curl} \mathbf{v} \in L^{2}(\Omega)^{3}\right\}
$$


We recall [13, Chap. I, Section 2] that the normal trace operator $\mathbf{v} \mapsto \mathbf{v} . \mathbf{n}$ is defined from $H(\operatorname{div}, \Omega)$ onto $H^{-1 / 2}(\partial \Omega)$ and the tangential trace operator $\mathbf{v} \mapsto \mathbf{v} \times \mathbf{n}$ is defined from $H(\operatorname{curl}, \Omega)$ into $H^{-1 / 2}(\partial \Omega)^{3}$. In view of the boundary conditions in system (2.1), we thus consider the spaces

$$
H_{0}(\operatorname{div}, \Omega)=\{\mathbf{v} \in H(\operatorname{div}, \Omega), \mathbf{v} \cdot \mathbf{n}=0 \text { on } \partial \Omega\}
$$

and

$$
H_{*}(\operatorname{curl}, \Omega)=\{\mathbf{v} \in H(\operatorname{curl}, \Omega), \mathbf{v} \times \mathbf{n}=\mathbf{0} \text { on } \Gamma\} .
$$

We set

$$
X(\Omega)=H_{0}(\operatorname{div}, \Omega) \cap H_{*}(\operatorname{curl}, \Omega)
$$

equipped with the semi-norm

$$
\|\mathbf{v}\|_{X(\Omega)}=\left(\|\operatorname{div} \mathbf{v}\|_{L^{2}(\Omega)}^{2}+\|\mathbf{c u r l} \mathbf{v}\|_{L^{2}(\Omega)^{3}}^{2}\right)^{1 / 2} .
$$

Since $\Omega$ is simply-connected, we recall from [2, Cor. 3.16] that this quantity is a norm, which is equivalent to the graph norm of $H(\operatorname{div}, \Omega) \cap H(\mathbf{c u r l}, \Omega)$, i.e., that there exists a constant $c$ only depending on $\Omega$ such that

$$
\forall \mathbf{v} \in X(\Omega), \quad\|\mathbf{v}\|_{L^{2}(\Omega)^{3}} \leq c|| \mathbf{v} \|_{X(\Omega)} .
$$

We denote by $L_{\circ}^{2}(\Omega)$ the space of functions in $L^{2}(\Omega)$ with a zero mean-value on $\Omega$, and we introduce the kernel

$$
V=\left\{\mathbf{v} \in X(\Omega) ; \forall q \in L_{\circ}^{2}(\Omega), \int_{\Omega} q(\mathbf{x}) \operatorname{div} \mathbf{v}(\mathbf{x}) d \mathbf{x}=0\right\},
$$

which is a closed subspace of $X(\Omega)$ and coincides with

$$
V=\{\mathbf{v} \in X(\Omega) ; \operatorname{div} \mathbf{v}=0 \text { in } \Omega\} .
$$

As usual, for handling time-dependent problems, it is convenient to consider functions defined on a time interval $] a, b\left[\right.$ with values in a separable functional space, say $Y$. More precisely, let $\|\cdot\|_{Y}$ denote the norm of $Y$; then for any $r, 1 \leq r \leq \infty$, we define

$$
L^{r}(a, b ; Y)=\{f \text { measurable in }] a, b\left[; \int_{a}^{b}\|f(\mathbf{t})\|_{Y}^{r} d t<\infty\right\},
$$

equipped with the norm

$$
\|f\|_{L^{r}(a, b ; Y)}=\left(\int_{a}^{b}\|f(\mathbf{t})\|_{Y}^{r} d t\right)^{1 / r},
$$

with the usual modifications if $r=\infty$. It is a Banach space if $Y$ is a Banach space.

We now assume that the data $\mathbf{f}$ belongs to $L^{2}\left(0, T ; X(\Omega)^{\prime}\right)$ where $X(\Omega)^{\prime}$ is the dual space of $X(\Omega)$, set $\mathbf{u}(t)=\mathbf{u}(t,$.$) and consider the following variational formulation in ] 0, T[$ : Find $\mathbf{u}(t)$ in $X(\Omega)$ and $p(t)$ in $L_{\circ}^{2}(\Omega)$ such that,

$$
\begin{gathered}
\forall \mathbf{v} \in X(\Omega), \quad\left(\frac{\partial}{\partial t} \mathbf{u}(t), \mathbf{v}\right)+\nu(\operatorname{curl} \mathbf{u}(t), \mathbf{c u r l} \mathbf{v})+(\operatorname{curl} \mathbf{u}(t) \times \mathbf{u}(t), \mathbf{v})-(\operatorname{div} \mathbf{v}, p(t))=\langle\mathbf{f}(t), \mathbf{v}\rangle, \\
\forall q \in L_{\circ}^{2}(\Omega), \quad(\operatorname{div} \mathbf{u}(t), q)=0, \\
\mathbf{u}(0)=\mathbf{0} .
\end{gathered}
$$

Proposition 2.1. Any solution of Problem (2.3) is a solution of Problem (2.1) where the first two equations are satisfied in the sense of distributions. Furthermore, every solution of (2.3) verify the bound

$$
\|\mathbf{u}\|_{L^{\infty}\left(0, T ; L^{2}(\Omega)^{3}\right)}^{2}+\frac{\nu}{2}\|\mathbf{u}\|_{L^{2}(0, T ; X(\Omega))}^{2} \leq \frac{1}{2 \nu}\|\mathbf{f}\|_{L^{2}\left(0, T ; X^{\prime}(\Omega)\right)}^{2}
$$


Proof. Let $(\mathbf{u}, p)$ be the solution of (2.3). Denoting by $\mathcal{D}(\Omega)$ the space of infinitely differentiable functions with a compact support in $\Omega$, we first take $\mathbf{v}$ in $\mathcal{D}(\Omega)^{3}$ in the first line of problem (2.3). This gives the first equation in problem (2.1). Next, it is readily checked from the Stokes formula that the second line of problem (2.3) is also satisfied when $q$ is a constant, hence for all $q$ in $L^{2}(\Omega)$. Thus, we take $q$ in $\mathcal{D}(\Omega)$, which yields the second equation in problem (2.1). It also follows from the definition of $X(\Omega)$ that the first two boundary conditions in problem (2.1) hold. Finally, introducing an infinitely differentiable function $\varphi$ with a compact support in $\Gamma_{m}$ and choosing $\mathbf{v}$ as a lifting in $X(\Omega) \cap H^{1}(\Omega)^{3}$ of the extension of $\boldsymbol{\varphi} \times \mathbf{n}$ by zero to $\partial \Omega$ gives the last boundary condition of problem (2.1).

To prove the bound (2.4), it suffices to take $\mathbf{v}=\mathbf{u}$ in (2.3), note that $(\operatorname{curl} \mathbf{u} \times \mathbf{u}, \mathbf{u})=0$ and integrate between 0 and $T$.

The spaces $L_{\circ}^{2}(\Omega)$ and $X(\Omega)$ verify a uniform inf-sup condition (see for instance [4] or [13, chap. I, Cor. $2.4])$ : There exists a constant $\beta_{*}>0$ such that

$$
\forall q \in L_{\circ}^{2}(\Omega), \quad \sup _{\mathbf{v} \in X(\Omega)} \frac{\int_{\Omega} q(\mathbf{x}) \operatorname{div} \mathbf{v}(\mathbf{x}) d \mathbf{x}}{\|\mathbf{v}\|_{X(\Omega)}} \geq \beta_{*}\|q\|_{L^{2}(\Omega)} .
$$

Remark 2.2. In the case when $\Omega$ has a $C^{1,1}$ boundary or is convex, it is proved in [2, Thm 2.17] that the space $H_{0}(\operatorname{div}, \Omega) \cap H(\mathbf{c u r l}, \Omega)$ is contained in $H^{1}(\Omega)^{3}$. We recall also that when $\Omega$ is a polyhedron, the space of restrictions of functions of $X(\Omega)$ to $\Omega \backslash \bar{\Theta}$, where $\Theta$ is a neighbourhood of the re-entrant corners of $\Omega$ inside $\Gamma_{m}$, is embedded in $H^{1}(\Omega \backslash \bar{\Theta})^{3}$ (see the proof of [4, Lemma 2.5] for more details). In any case, we are led to make the following assumption to give a sense to the nonlinear term in Problem (2.3).

Assumption 2.3. The domain $\Omega$ has a $C^{1,1}$ boundary or is a polyhedron with no re-entrant corners inside $\Gamma_{m}$.

When this assumption is satisfied, the arguments for the proof of the existence of a solution to Problem (2.3) are exactly the same as [16, chap. III, Theorem 1.1], see also [12, chap. V]. We prefer to omit this proof since it is beyong the aim of this paper.

\section{The DisCRETE PROBLEM}

From now on, we assume that $\Omega$ is a polyhedron and that $\mathbf{f}$ belongs to $\mathcal{C}^{0}\left(0, T ; X(\Omega)^{\prime}\right)$. In order to describe the time discretization with an adaptive choice of local time steps, we introduce a partition of the interval $[0, T]$ into subintervals $\left[t_{n-1}, t_{n}\right], 1 \leq n \leq N$, such that $0=t_{0}<t_{1}<\cdots<t_{N}=T$. We denote by $\tau_{n}$ the length of $\left[t_{n-1}, t_{n}\right]$, by $\tau$ the N-tuple $\left(\tau_{1}, \ldots, \tau_{N}\right)$, by $|\tau|$ the maximum of the $\tau_{n}$, $1 \leq n \leq N$, and finally by $\sigma_{\tau}$ the regularity parameter

$$
\sigma_{\tau}=\max _{2 \leq n \leq N} \frac{\tau_{n}}{\tau_{n-1}} .
$$

In what follows, we work with a regular family of partitions, i.e. we assume that $\sigma_{\tau}$ is bounded independently of $\tau$.

We introduce the operator $\pi_{\tau}$ (resp. $\pi_{l, \tau}$ ): For any Banach space $X$ and any function $g$ continuous from ] $0, T]$ (resp. $\left[0, T\left[\right.\right.$ ) into $X, \pi_{\tau} g$ (resp. $\pi_{l, \tau} g$ ) denotes the step function which is constant and equal to $g\left(t_{n}\right)$ (resp. $g\left(t_{n-1}\right)$ ) on each interval $\left.] t_{n-1}, t_{n}\right], 1 \leq n \leq N$. Similarly, with any sequence $\left(\phi_{n}\right)_{0 \leq n \leq N}$ in $X$, we associate the step function $\pi_{\tau} \phi_{\tau}$ (resp. $\pi_{l, \tau} \phi_{\tau}$ ) which is constant and equal to $\phi_{n}$ (resp. $\phi_{n-1}$ ) on each interval $\left.] t_{n-1}, t_{n}\right], 1 \leq n \leq N$.

Furthermore, for any Banach space $X$, with each family $\left(\mathbf{v}_{n}\right)_{0 \leq n \leq N}$ in $X^{N+1}$, we agree to associate the function $\mathbf{v}_{\tau}$ on $[0, T]$ which is affine on each interval $\left[t_{n-1}, t_{n}\right], 1 \leq n \leq N$, and equal to $\mathbf{v}_{n}$ at $t_{n}$, $0 \leq n \leq N$.

We now describe the space discretization. For each $n, 0 \leq n \leq N$, let $\left(\mathcal{T}_{n h}\right)_{h}$ be a regular family of triangulations of $\Omega$ by tetrahedra, in the usual sense that:

- for each $h, \bar{\Omega}$ is the union of all elements of $\mathcal{T}_{n h}$;

- the intersection of two different elements of $\mathcal{T}_{n h}$, if not empty, is a vertex or a whole edge or a whole face of both of them; 
- the ratio of the diameter of an element $K$ in $\mathcal{T}_{n h}$ to the diameter of its inscribed sphere is bounded by a constant independent of $n$ and $h$.

As usual, $h$ denotes the maximal diameter of the elements of all $\mathcal{T}_{n h}, 0 \leq n \leq N$, while for each $n, h_{n}$ denotes the maximal diameter of the elements of $\mathcal{T}_{n h}$. For each $\kappa$ in $\mathcal{T}_{n h}$ and each nonnegative integer $k$, we denote by $P_{k}(\kappa)$ the space of restrictions to $\kappa$ of polynomials with 3 variables and total degree at most $k$.

In what follows, $c, c^{\prime}, C, C^{\prime}, c_{1}, \ldots$ stand for generic constants which may vary from line to line but are always independent of $h$ and $n$. From now on, we call finite element space associated with $\mathcal{T}_{n h}$ a space of functions such that their restrictions to any element $\kappa$ of $\mathcal{T}_{n h}$ belong to a space of polynomials of fixed degree.

For each $n$ and $h$, we associate with $\mathcal{T}_{n h}$ two finite element spaces $X_{n h}$ and $M_{n h}$ which are contained in $X(\Omega)$ and $L_{\circ}^{2}(\Omega)$, respectively, and such that the following inf-sup condition holds for a constant $\beta>0$ :

$$
\forall q_{h} \in M_{n h}, \quad \sup _{\mathbf{v}_{h} \in X_{n h}} \frac{\int_{\Omega} q_{h}(\mathbf{x}) \operatorname{div} \mathbf{v}_{h}(\mathbf{x}) d \mathbf{x}}{\left\|\mathbf{v}_{h}\right\|_{X(\Omega)}} \geq \beta\left\|q_{h} \mid\right\|_{L^{2}(\Omega)} .
$$

Indeed, there exist many examples of finite element spaces satisfying these conditions (the inf-sup condition being usually proved with $X_{n h}$ replaced by $\left.X_{n h} \cap H_{0}^{1}(\Omega)^{d}\right)$, see [13, chap. II]. We give one example of them dealing with continuous discrete pressures which is presented in [13, chap. II, section 4.1] for instance. The velocity is discretized with the "Mini-Element"

$$
X_{n h}=\left\{\mathbf{v}_{h} \in X(\Omega) ; \forall \kappa \in \mathcal{T}_{n h},\left.\mathbf{v}_{h}\right|_{\kappa} \in P_{b}(\kappa)^{3}\right\},
$$

where the space $P_{b}(\kappa)$ is spanned by functions in $P_{1}(\kappa)$ and the bubble function on $\kappa$ (for each element $\kappa$, the bubble function is equal to the product of the barycentric coordinates associated with the vertices of $\kappa$ ). The pressure is discretized with classical continuous finite elements of order one

$$
M_{n h}=\left\{q_{h} \in L_{\circ}^{2}(\Omega) \cap H^{1}(\Omega) ; \forall \kappa \in \mathcal{T}_{n h},\left.q_{h}\right|_{\kappa} \in P_{1}(\kappa)\right\} .
$$

As usual, we denote by $V_{n h}$ the kernel

$$
V_{n h}=\left\{\mathbf{v}_{h} \in X_{n h} ; \forall q_{h} \in M_{n h}, \int_{\Omega} q_{h}(\mathbf{x}) \operatorname{div} \mathbf{v}_{h}(\mathbf{x}) d \mathbf{x}=0\right\} .
$$

The discrete problem associated with Problem (2.3) is: Knowing $\mathbf{u}_{h}^{n-1} \in X_{n-1 h}$, find $\left(\mathbf{u}_{h}^{n}, p_{h}^{n}\right)$ with values in $X_{n h} \times M_{n h}$ solution of

$$
\begin{gathered}
\forall \mathbf{v}_{h} \in X_{n h}, \quad \frac{1}{\tau_{n}}\left(\mathbf{u}_{h}^{n}-\mathbf{u}_{h}^{n-1}, \mathbf{v}_{h}\right)+\nu\left(\operatorname{curl} \mathbf{u}_{h}^{n}, \operatorname{curl} \mathbf{v}_{h}\right)+\nu\left(\operatorname{div} \mathbf{u}_{h}^{n}, \operatorname{div} \mathbf{v}_{h}\right) \\
+\left(\operatorname{curl} \mathbf{u}_{h}^{n-1} \times \mathbf{u}_{h}^{n}, \mathbf{v}_{h}\right)-\left(p_{h}^{n}, \operatorname{div} \mathbf{v}_{h}\right)=\left\langle\mathbf{f}^{n}, \mathbf{v}_{h}\right\rangle \\
\forall q_{h} \in M_{n h}, \quad\left(\operatorname{div} \mathbf{u}_{h}^{n}, q_{h}\right)=0
\end{gathered}
$$

by assuming that $\mathbf{u}_{h}^{0}=\mathbf{0}$ and taking

$$
\mathbf{f}^{n}(\mathbf{x})=\mathbf{f}\left(\mathbf{x}, t_{n}\right), \quad \text { for a.e. } \mathbf{x} \text { in } \Omega .
$$

We begin by showing a bound for the solution $\mathbf{u}_{h}^{n}$ of Problem (3.2) - (3.3).

Theorem 3.1. At each time step, knowing $\mathbf{u}_{h}^{n-1} \in X_{n-1 h}$, Problem (3.2)-(3.3) admits a unique solution $\left(\mathbf{u}_{h}^{n}, p_{h}^{n}\right)$ with values in $X_{n h} \times M_{n h}$. This solution satisfies, for $m=1, \ldots, N$,

$$
\frac{1}{2}\left\|\mathbf{u}_{h}^{m}\right\|_{L^{2}(\Omega)^{3}}^{2}+\frac{\nu}{2} \sum_{n=1}^{m} \tau_{n}\left\|\mathbf{u}_{h}^{n}\right\|_{X(\Omega)}^{2} \leq \frac{c^{2}}{\nu}\left\|\pi_{\tau} \mathbf{f}\right\|_{L^{2}\left(0, T ; X(\Omega)^{\prime}\right)}^{2} \leq \frac{c^{\prime 2}}{\nu}\|\mathbf{f}\|_{L^{\infty}\left(0, T ; X(\Omega)^{\prime}\right)}^{2} .
$$

Proof. For $\mathbf{u}_{h}^{n-1} \in X_{n-1 h}$, it is clear that Problem (3.2) - (3.3) has a unique solution $\left(\mathbf{u}_{h}^{n}, p_{h}^{n}\right)$ as a consequence of the coerciveness of the corresponding bilinear form on $X_{n h} \times X_{n h}$ and the inf-sup condition (3.1). Therefore, we take $\mathbf{v}_{h}=\mathbf{u}_{h}^{n}$ in (3.2) and we use the relation

$$
a(a-b)=\frac{1}{2} a^{2}+\frac{1}{2}(a-b)^{2}-\frac{1}{2} b^{2},
$$


and inequality $(2.2)$ to obtain the relation :

$$
\frac{1}{2}\left\|\mathbf{u}_{h}^{n}\right\|_{L^{2}(\Omega)^{d}}^{2}-\frac{1}{2}\left\|\mathbf{u}_{h}^{n-1}\right\|_{L^{2}(\Omega)^{d}}^{2}+\nu \tau_{n}\left\|\mathbf{u}_{h}^{n}\right\|_{X(\Omega)}^{2} \leq \frac{\tau_{n} \varepsilon}{2}\left\|\mathbf{f}^{n}\right\|_{X(\Omega)^{\prime}}^{2}+\frac{\tau_{n} c^{2}}{2 \varepsilon}\left\|\mathbf{u}_{h}^{n}\right\|_{X(\Omega)}^{2} .
$$

We choose $\varepsilon=\frac{c^{2}}{\nu}$ and sum over $n=1, \ldots m$. We obtain :

$$
\frac{1}{2}\left\|\mathbf{u}_{h}^{m}\right\|_{L^{2}(\Omega)^{d}}^{2}+\frac{\nu}{2} \sum_{n=1}^{m} \tau_{n}\left\|\mathbf{u}_{h}^{n}\right\|_{X(\Omega)}^{2} \leq \sum_{n=1}^{m} \frac{\tau_{n} c^{2}}{2 \nu}\left\|\mathbf{f}^{n}\right\|_{X(\Omega)^{\prime}}^{2}
$$

This implies the estimates.

\section{A POSTERIORI ERROR ANALYSIS}

We now intend to prove a posteriori error estimates between the exact solution $(\mathbf{u}, p)$ of Problem (2.3) and the numerical solution $\left(\mathbf{u}_{h}^{n}, p_{h}^{n}\right)$ of Problem $(3.2)-(3.3)$. Several steps are needed for that.

4.1. Construction of the error indicators. We first introduce the space

$$
Z_{n h}=\left\{\mathbf{g}_{h} \in L^{2}(\Omega)^{d} ; \forall \kappa \in \mathcal{T}_{n h},\left.\mathbf{g}_{h}\right|_{\kappa} \in P_{\ell}(\kappa)^{d}\right\},
$$

where $\ell$ is usually lower than the maximal degree of polynomials in $X_{n h}$, and, for $1 \leq n \leq N$, we fix an approximation $\mathbf{f}_{h}^{n}$ of the data $\mathbf{f}^{n}$ in $Z_{n h}$.

Next, for every element $\kappa$ in $\mathcal{T}_{n h}$, we denote by

- $\varepsilon_{\kappa}$ the set of faces of $\kappa$ that are not contained in $\partial \Omega$,

- $\varepsilon_{\kappa}^{m}$ the set of faces of $\kappa$ which are contained in $\bar{\Gamma}_{m}$,

- $\Delta_{\kappa}$ the union of elements of $\mathcal{T}_{n h}$ that intersect $\kappa$,

- $\Delta_{e}$ the union of elements of $\mathcal{T}_{n h}$ that intersect the face $e$,

- $h_{\kappa}$ the diameter of $\kappa$ and $h_{e}$ the diameter of the face $e$,

- and $[\cdot]_{e}$ the jump through $e$ for each face $e$ in an $\varepsilon_{\kappa}$ (making its sign precise is not necessary).

Also, $\mathbf{n}_{\kappa}$ stands for the unit outward normal vector to $\kappa$ on $\partial \kappa$.

For the demonstration of the next theorems, we introduce for an element $\kappa$ of $\mathcal{T}_{n h}$, the bubble function $\psi_{\kappa}$ (resp. $\psi_{e}$ for the face $e$ ) which is equal to the product of the 4 barycentric coordinates associated with the vertices of $\kappa$ (resp. of the 3 barycentric coordinates associated with the vertices of $e$ ). We also consider a lifting operator $\mathcal{L}_{e}$ defined on polynomials on $e$ vanishing on $\partial e$ into polynomials on the at most two elements $\kappa$ containing $e$ and vanishing on $\partial \kappa \backslash e$, which is constructed by affine transformation from a fixed operator on the reference element. We recall the next results from [17, Lemma 3.3].

Property 4.1. Denoting by $P_{r}(\kappa)$ the space of polynomials of degree smaller than $r$ on $\kappa$, we have

$$
\forall v \in P_{r}(\kappa), \quad\left\{\begin{array}{l}
c\|v\|_{0, \kappa} \leq\left\|v \psi_{\kappa}^{1 / 2}\right\|_{0, \kappa} \leq c^{\prime}\|v\|_{0, \kappa}, \\
|v|_{1, \kappa} \leq c h_{\kappa}^{-1}\|v\|_{0, \kappa} .
\end{array}\right.
$$

Property 4.2. Denoting by $P_{r}(e)$ the space of polynomials of degree smaller than $r$ on $e$, we have

$$
\forall v \in P_{r}(e), \quad c\|v\|_{0, e} \leq\left\|v \psi_{e}^{1 / 2}\right\|_{0, e} \leq c^{\prime}\|v\|_{0, e},
$$

and, for all polynomials $v$ in $P_{r}(e)$ vanishing on $\partial e$, if $\kappa$ is an element which contains $e$,

$$
\left\|\mathcal{L}_{e} v\right\|_{0, \kappa}+h_{e}\left|\mathcal{L}_{e} v\right|_{1, \kappa} \leq c h_{e}^{1 / 2}\|v\|_{0, e} .
$$

We also introduce a Clément type regularization operator $\mathcal{C}_{n h}[9]$ which has the following properties, see [5, section IX.3]: For any function $\mathbf{w}$ in $H^{1}(\Omega)^{d}, \mathcal{C}_{n h} \mathbf{w}$ belongs to the continuous affine finite element space and satisfies for any $\kappa$ in $\mathcal{T}_{n h}$ and $e$ in $\varepsilon_{\kappa}$,

$$
\left\|\mathbf{w}-\mathcal{C}_{n h} \mathbf{w}\right\|_{0, \kappa} \leq c h_{\kappa}\|\mathbf{w}\|_{1, \Delta_{\kappa}} \quad \text { and } \quad\left\|\mathbf{w}-\mathcal{C}_{n h} \mathbf{w}\right\|_{0, e} \leq c h_{e}^{1 / 2}\|\mathbf{w}\|_{1, \Delta_{e}} .
$$


For the a posteriori error studies, in order to uncouple the time and space errors, we introduce the following time semi-discrete problem: Knowing $\mathbf{u}^{n-1}$ in $X(\Omega)$, find $\left(\mathbf{u}^{n}, p^{n}\right)$ with values in $X(\Omega) \times L_{\circ}^{2}(\Omega)$ solution of

$$
\begin{aligned}
& \forall \mathbf{v} \in X(\Omega), \quad \frac{1}{\tau_{n}}\left(\mathbf{u}^{n}-\mathbf{u}^{n-1}, \mathbf{v}\right)+\nu\left(\operatorname{curl} \mathbf{u}^{n}, \mathbf{c u r l} \mathbf{v}\right)+\nu\left(\operatorname{div} \mathbf{u}^{n}, \operatorname{div} \mathbf{v}\right) \\
& -\left(\operatorname{div} \mathbf{v}, p^{n}\right)=\left\langle\tilde{\mathbf{f}}\left(t_{n}\right), \mathbf{v}\right\rangle, \\
& \forall q \in L_{\circ}^{2}(\Omega), \quad\left(\operatorname{div} \mathbf{u}^{n}, q\right)=0,
\end{aligned}
$$

by assuming that $\mathbf{u}^{0}=\mathbf{0}$ and taking $\tilde{\mathbf{f}}\left(t_{n}\right)=\mathbf{f}\left(t_{n}\right)-\operatorname{curl} \mathbf{u}^{n-1} \times \mathbf{u}^{n}$. It is clear that Problem (4.3)- (4.4) has a unique solution owing to the ellipticity of the bilinear form and the inf-sup condition on the form for the divergence. We consider the piecewise affine function $\mathbf{u}_{\tau}$ which take in the interval $\left[t_{n-1}, t_{n}\right]$ the values

$$
\mathbf{u}_{\tau}(t)=\frac{t-t_{n-1}}{\tau_{n}}\left(\mathbf{u}^{n}-\mathbf{u}^{n-1}\right)+\mathbf{u}^{n-1} .
$$

All this leads to the first residual equation: Since the solution of problem (2.3) is divergence-free, the solutions of Problems (2.3) and (4.3) - (4.4) verify for $t$ in $\left.] t_{n-1}, t_{n}\right]$ and for all $\mathbf{v}(t)$ in $X(\Omega)$,

$$
\begin{gathered}
\left(\frac{\partial}{\partial t}\left(\mathbf{u}-\mathbf{u}_{\tau}\right)(t), \mathbf{v}(t)\right)+\nu\left(\operatorname{curl}\left(\mathbf{u}(t)-\mathbf{u}_{\tau}(t)\right), \operatorname{curl} \mathbf{v}(t)\right)+\nu\left(\operatorname{div}\left(\mathbf{u}(t)-\mathbf{u}_{\tau}(t)\right), \operatorname{div} \mathbf{v}(t)\right) \\
+\left(\operatorname{curl} \mathbf{u}(t) \times \mathbf{u}(t)-\operatorname{curl} \mathbf{u}_{\tau}(t) \times \mathbf{u}_{\tau}(t), \mathbf{v}(t)\right)-\left(\operatorname{div} \mathbf{v}(t), p(t)-\pi_{\tau} p_{\tau}(t)\right) \\
=(\mathbf{f}(t), \mathbf{v}(t))-\frac{1}{\tau_{n}}\left(\mathbf{u}^{n}-\mathbf{u}^{n-1}, \mathbf{v}(t)\right)-\nu\left(\mathbf{c u r l} \mathbf{u}_{\tau}(t), \operatorname{curl} \mathbf{v}(t)\right)-\nu\left(\operatorname{div} \mathbf{u}_{\tau}(t), \operatorname{div} \mathbf{v}(t)\right) \\
-\left(\operatorname{curl} \mathbf{u}_{\tau}(t) \times \mathbf{u}_{\tau}(t), \mathbf{v}(t)\right)+\left(\operatorname{div} \mathbf{v}(t), \pi_{\tau} p_{\tau}(t)\right),
\end{gathered}
$$

and for all $q(t)$ in $L_{\circ}^{2}(\Omega)$,

$$
\int_{\Omega} q(t, \mathbf{x}) \operatorname{div}\left(\mathbf{u}(t, \mathbf{x})-\mathbf{u}_{\tau}(t, \mathbf{x})\right) d \mathbf{x}=-\int_{\Omega} q(t, \mathbf{x}) \operatorname{div} \mathbf{u}_{\tau}(t, \mathbf{x}) d \mathbf{x}=0 .
$$

The right-hand side of equation (4.6) can still be written $\left\langle\mathbf{f}-\mathbf{f}^{n}+R^{\tau}\left(\mathbf{u}_{\tau}\right), \mathbf{v}(t)\right\rangle$, where the residual $R^{\tau}\left(\mathbf{u}_{\tau}\right)$ is given by

$$
\begin{aligned}
\left\langle R^{\tau}\left(\mathbf{u}_{\tau}\right)(t), \mathbf{v}(t)\right\rangle=\nu\left(\operatorname{curl}\left(\mathbf{u}^{n}-\mathbf{u}_{\tau}(t)\right), \operatorname{curl} \mathbf{v}(t)\right)+ & \nu\left(\operatorname{div}\left(\mathbf{u}^{n}-\mathbf{u}_{\tau}(t)\right), \operatorname{div} \mathbf{v}(t)\right) \\
& +\left(\operatorname{curl} \mathbf{u}^{n-1} \times \mathbf{u}^{n}-\operatorname{curl} \mathbf{u}_{\tau}(t) \times \mathbf{u}_{\tau}(t), \mathbf{v}(t)\right) .
\end{aligned}
$$

By using (4.5), this yields

$$
\begin{gathered}
\left\langle R^{\tau}\left(\mathbf{u}_{\tau}\right)(t), \mathbf{v}(t)\right\rangle=\frac{t_{n}-t}{\tau_{n}} \sum_{\kappa \in \mathcal{T}_{n h}}\left\{\nu \int_{\kappa} \operatorname{curl}\left(\mathbf{u}^{n}-\mathbf{u}^{n-1}\right)(\mathbf{x}) \cdot \operatorname{curl} \mathbf{v}(t, \mathbf{x}) d \mathbf{x}\right. \\
+\nu \int_{\kappa} \operatorname{div}\left(\mathbf{u}^{n}-\mathbf{u}^{n-1}\right)(\mathbf{x}) \cdot \operatorname{div} \mathbf{v}(t, \mathbf{x}) d \mathbf{x}+\int_{\kappa}\left(\operatorname{curl} \mathbf{u}^{n-1} \times\left(\mathbf{u}^{n}-\mathbf{u}^{n-1}\right) \mathbf{v}(t, \mathbf{x}) d \mathbf{x}\right\} \\
\quad-\frac{t-t_{n-1}}{\tau_{n}} \sum_{\kappa \in \mathcal{T}_{n h}} \int_{\kappa} \operatorname{curl}\left(\mathbf{u}^{n}-\mathbf{u}^{n-1}\right) \times \mathbf{u}_{\tau}(t, \mathbf{x}) \mathbf{v}(t, \mathbf{x}) d \mathbf{x} .
\end{gathered}
$$

On the other hand, we consider the piecewise affine function $\mathbf{u}_{h}$ which takes in the interval $\left[t_{n-1}, t_{n}\right]$ the values

$$
\mathbf{u}_{h}(t)=\frac{t-t_{n-1}}{\tau_{n}}\left(\mathbf{u}_{h}^{n}-\mathbf{u}_{h}^{n-1}\right)+\mathbf{u}_{h}^{n-1} .
$$


We now write the second residual equation: The solutions of Problems (4.3) - (4.4) and (3.2) - (3.3) verify for $t$ in $\left.] t_{n-1}, t_{n}\right]$ and for all $\mathbf{v}(t)$ in $X(\Omega)$,

$$
\begin{aligned}
& \left(\frac{\partial}{\partial t}\left(\mathbf{u}_{\tau}-\mathbf{u}_{h}\right)(t), \mathbf{v}(t)\right)+\nu\left(\operatorname{curl}\left(\mathbf{u}_{\tau}(t)-\mathbf{u}_{h}(t)\right), \operatorname{curl} \mathbf{v}(t)\right)+\nu\left(\operatorname{div}\left(\mathbf{u}_{\tau}(t)-\mathbf{u}_{h}(t)\right), \operatorname{div} \mathbf{v}(t)\right) \\
& +\left(\operatorname{curl} \pi_{l, \tau} \mathbf{u}_{\tau}(t) \times \pi_{\tau} \mathbf{u}_{\tau}(t)-\operatorname{curl} \pi_{l, \tau} \mathbf{u}_{h}(t) \times \pi_{\tau} \mathbf{u}_{h}(t), \mathbf{v}(t)\right)-\left(\operatorname{div} \mathbf{v}(t), p(t)-\pi_{\tau} p_{\tau}(t)\right) \\
& =\left(\mathbf{f}^{n}(t), \mathbf{v}(t)\right)-\frac{1}{\tau_{n}}\left(\mathbf{u}_{h}^{n}-\mathbf{u}_{h}^{n-1}, \mathbf{v}(t)\right)-\nu\left(\operatorname{curl} \mathbf{u}_{h}(t), \operatorname{curl} \mathbf{v}(t)\right)-\nu\left(\operatorname{div} \mathbf{u}_{h}(t), \operatorname{div} \mathbf{v}(t)\right) \\
& -\left(\operatorname{curl} \pi_{l, \tau} \mathbf{u}_{h}(t) \times \pi_{\tau} \mathbf{u}_{h}(t), \mathbf{v}(t)\right)+\left(\operatorname{div} \mathbf{v}(t), \pi_{\tau} p_{\tau}(t)\right),
\end{aligned}
$$

and for all $q(t)$ in $L_{\circ}^{2}(\Omega)$,

$$
\int_{\Omega} q(t, \mathbf{x}) \operatorname{div}\left(\mathbf{u}(t, \mathbf{x})-\mathbf{u}_{h}(t, \mathbf{x})\right) d \mathbf{x}=-\int_{\Omega} q(t, \mathbf{x}) \operatorname{div} \mathbf{u}_{h}(t, \mathbf{x}) d \mathbf{x}
$$

Thus, the right-hand side of equation (4.10) can be written $\left\langle\mathbf{f}^{n}-\mathbf{f}_{h}^{n}+R^{h}\left(\mathbf{u}_{h}\right), \mathbf{v}-\mathbf{v}_{\mathbf{h}}\right\rangle$, where the residual $R^{h}\left(\mathbf{u}_{h}\right)$ is given in $L^{2}\left(0, T ; X(\Omega)^{\prime}\right)$ by, for $t$ in $\left.] t_{n-1}, t_{n}\right]$ and for all $\mathbf{v}(t)$ in $X(\Omega)$ and any approximation $\mathbf{v}_{h}(t)$ of $\mathbf{v}(t)$ in $X_{h}$

$$
\begin{aligned}
& \left\langle R^{h}\left(\mathbf{u}_{h}\right)(t), \mathbf{v}(t)-\mathbf{v}_{h}(t)\right\rangle=\left(\mathbf{f}_{h}^{n}-\frac{1}{\tau_{n}}\left(\mathbf{u}_{h}^{n}-\mathbf{u}_{h}^{n-1}\right), \mathbf{v}(t)-\mathbf{v}_{h}(t)\right)+\left(\operatorname{div}\left(\mathbf{v}(t)-\mathbf{v}_{h}(t)\right), p_{h}^{n}\right) \\
& \quad-\left(\operatorname{curl} \mathbf{u}_{h}^{n-1} \times \mathbf{u}_{h}^{n}, \mathbf{v}(t)-\mathbf{v}_{h}(t)\right)-\nu\left(\operatorname{curl} \mathbf{u}_{h}^{n}, \operatorname{curl}\left(\mathbf{v}(t)-\mathbf{v}_{h}(t)\right)\right)-\nu\left(\operatorname{div} \mathbf{u}_{h}^{n}, \operatorname{div}\left(\mathbf{v}(t)-\mathbf{v}_{h}(t)\right)\right) .
\end{aligned}
$$

By integration by parts on each $\kappa$ in $\mathcal{T}_{n h}$, it can equivalently be written

$$
\begin{gathered}
\left\langle R^{h}\left(\mathbf{u}_{h}\right)(t), \mathbf{v}(t)-\mathbf{v}_{h}(t)\right\rangle \\
=\sum_{\kappa \in \mathcal{T}_{n h}}\left\{\int _ { \kappa } \left(\mathbf{f}_{h}^{n}-\frac{1}{\tau_{n}}\left(\mathbf{u}_{h}^{n}-\mathbf{u}_{h}^{n-1}\right)-\nu \operatorname{curl} \mathbf{c u r l} \mathbf{u}_{h}^{n}+\nu \nabla \operatorname{div} \mathbf{u}_{h}^{n}-\operatorname{curl} \mathbf{u}_{h}^{n-1} \times \mathbf{u}_{h}^{n}\right.\right. \\
\left.-\nabla p_{h}^{n}\right)(\mathbf{x}) \cdot\left(\mathbf{v}(t, \mathbf{x})-\mathbf{v}_{h}(t, \mathbf{x})\right) d \mathbf{x} \\
-\sum_{e \in \varepsilon_{\kappa}} \int_{e}\left(\nu \operatorname{curl} \mathbf{u}_{h}^{n} \times \mathbf{n}+\nu\left(\operatorname{div} \mathbf{u}_{h}^{n}\right) \mathbf{n}-p_{h}^{n} \mathbf{n}\right)(\boldsymbol{\sigma}) \cdot\left(\mathbf{v}(t, \boldsymbol{\sigma})-\mathbf{v}_{h}(t, \boldsymbol{\sigma})\right) d \boldsymbol{\sigma} \\
\left.-\nu \sum_{e \in \varepsilon_{\kappa}^{m}} \int_{e}\left(\operatorname{curl} \mathbf{u}_{h}^{n} \times \mathbf{n}\right)(\boldsymbol{\sigma}) \cdot\left(\mathbf{v}(t, \boldsymbol{\sigma})-\mathbf{v}_{h}(t, \boldsymbol{\sigma})\right) d \boldsymbol{\sigma}\right\}
\end{gathered}
$$

(where $\boldsymbol{\sigma}$ stands for the tangential coordinates on $e$ ).

All this leads to the following definition of the error indicators: For each $\kappa$ in $\mathcal{T}_{n h}$,

$$
\begin{gathered}
\left(\eta_{n, \kappa}^{\tau}\right)^{2}=\tau_{n}\left\|\mathbf{u}_{h}^{n}-\mathbf{u}_{h}^{n-1}\right\|_{X(\Omega)}^{2} \\
\left(\eta_{n, \kappa}^{h}\right)^{2}=h_{\kappa}^{2}\left\|\mathbf{f}_{h}^{n}-\frac{1}{\tau_{n}}\left(\mathbf{u}_{h}^{n}-\mathbf{u}_{h}^{n-1}\right)-\nu \operatorname{curl} \operatorname{curl} \mathbf{u}_{h}^{n}+\nu \nabla \operatorname{div} \mathbf{u}_{h}^{n}-\operatorname{curl} \mathbf{u}_{h}^{n-1} \times \mathbf{u}_{h}^{n}-\nabla p_{h}^{n}\right\|_{0, \kappa}^{2} \\
+\left\|\operatorname{div} \mathbf{u}_{h}^{n}\right\|_{0, \kappa}^{2}+\sum_{e \in \varepsilon_{\kappa}} h_{e}\left\|\left[\nu \operatorname{curl} \mathbf{u}_{h}^{n} \times \mathbf{n}+\nu\left(\operatorname{div} \mathbf{u}_{h}^{n}\right) \mathbf{n}-p_{h}^{n} \mathbf{n}\right]_{e}\right\|_{0, e}^{2}+\sum_{e \in \varepsilon_{\kappa}^{m}} h_{e}\left\|\nu \operatorname{curl} \mathbf{u}_{h}^{n} \times \mathbf{n}\right\|_{0, e}^{2}
\end{gathered}
$$

Even if these indicators are a little complex, each term in them is easy to compute since it only depends on the discrete solution and involves (usually low degree) polynomials. The following lemma justifies our choice of error indicators.

Lemma 4.3. When $\Omega$ has no re-entrant corner inside $\Gamma_{m}$, the following estimates hold for $1 \leq n \leq N$,

(1) For all $\mathbf{v}$ in $X(\Omega)$ and $t$ in $\left.] t_{n-1}, t_{n}\right]$,

$$
\left\langle R^{\tau}\left(\mathbf{u}_{\tau}\right)(t), \mathbf{v}\right\rangle \leq C \frac{t_{n}-t}{\tau_{n}^{3 / 2}}\left(\sum_{\kappa \in \mathcal{T}_{n h}}\left(\left(\eta_{n, \kappa}^{\tau}\right)^{2}+\sum_{k=n-1}^{n} \tau_{k}\left\|\mathbf{u}^{k}-\mathbf{u}_{h}^{k}\right\|_{X(\Omega)}^{2}\right)^{1 / 2}\|\mathbf{v}\|_{X(\Omega)} .\right.
$$

(2) For all $\mathbf{v}$ in $X(\Omega)$ and $\mathbf{v}_{h}=\mathcal{C}_{n h} \mathbf{v}$ :

$$
\left\langle R^{h}\left(\mathbf{u}_{h}\right), \mathbf{v}-\mathbf{v}_{h}\right\rangle \leq C\left(\sum_{\kappa \in \mathcal{T}_{n h}}\left(\eta_{n, \kappa}^{h}\right)^{2}\right)^{1 / 2}\|\mathbf{v}\|_{X(\Omega)} .
$$


Proof. We proceed in two steps, one for each estimate.

1) From equation (4.8), by using a Cauchy-Schwarz inequality and noting that both $\mathbf{c u r l}^{n-1}$ and $\mathbf{u}_{\tau}$ are bounded in appropriate norms (this is proved like Theorem 3.1), we derive

$$
\left\langle R^{\tau}\left(\mathbf{u}_{\tau}\right)(t), \mathbf{v}\right\rangle \leq C \frac{t_{n}-t}{\tau_{n}^{3 / 2}}\left(\sum_{\kappa \in \mathcal{T}_{n h}} \tau_{n}\left\|\mathbf{u}^{n}-\mathbf{u}^{n-1}\right\|_{X(\Omega)}^{2}\right)^{1 / 2}\|\mathbf{v}\|_{X(\Omega)} .
$$

Next, we use the triangle inequality

$$
\tau_{n}^{\frac{1}{2}}\left\|\mathbf{u}^{n}-\mathbf{u}^{n-1}\right\|_{X(\Omega)} \leq \tau_{n}^{\frac{1}{2}}\left\|\mathbf{u}_{h}^{n}-\mathbf{u}_{h}^{n-1}\right\|_{X(\Omega)}+\tau_{n}^{\frac{1}{2}}\left\|\mathbf{u}^{n}-\mathbf{u}_{h}^{n}\right\|_{X(\Omega)}+\tau_{n}^{\frac{1}{2}}\left\|\mathbf{u}^{n-1}-\mathbf{u}_{h}^{n-1}\right\|_{X(\Omega)},
$$

and we conclude by using the regularity of the family of partitions.

2) We derive the result from formula (4.12) with $\mathbf{v}_{h}=\mathcal{C}_{n h} \mathbf{v}$, by using the Cauchy-Schwarz inequality, the proprties of $\mathcal{C}_{n h}$ and the continuity of the imbedding of $X(\Omega)$ in $H^{1}(\Omega)$.

4.2. Upper bounds of the error. To prove the upper bound, we follow the idea used by C. Bernardi and R. Verfürth in [8] (or C. Bernardi and T. Sayah in [6]) in order to uncouple time and space errors. We calculate upper bounds for the errors between the solution of problem $(4.3)-(4.4)$ and the exact solution firstly and the discrete solution secondly, by using the theory of Pousin and Rappaz [15] to handle the nonlinear term.

We denote by $\mathcal{S}$ the operator which associates with any data $\mathbf{f}$ in $L^{2}\left(0, T ; X(\Omega)^{\prime}\right)$ the part $\mathbf{u}$ in the space $L^{\infty}\left(0, T ; L^{2}(\Omega)^{d}\right) \cap L^{2}(0, T ; X(\Omega))$ of the solution of Problem $(2.3)$ in the linear case, i.e. when suppressing the term $(\mathbf{c u r l} \mathbf{u}(t) \times \mathbf{u}(t), \mathbf{v})$. We refer to [6, Proposition 2.2] for the main properties of the operator $\mathcal{S}$. We also set: $\mathbf{f}^{*}(\mathbf{u})=\mathbf{f}-\mathbf{c u r l} \mathbf{u} \times \mathbf{u}$ and denote by $D$ the differential operator with respect to $\mathbf{u}$.

Theorem 4.4. Let $\mathbf{u}$ be a solution of Problem (2.3) such that $\operatorname{Id}-\mathcal{S} D \mathbf{f}^{*}(\mathbf{u})$ is an isomorphism of $L^{2}(0, T ; X(\Omega))$. When $\Omega$ has no re-entrant corner inside $\Gamma_{m}$, there exists a neighbourhood of $\mathbf{u}$ in $L^{2}(0, T ; X(\Omega))$ such that the following bound holds for any velocity $\mathbf{u}_{\tau}$ associated with the solutions $\left(\mathbf{u}^{n}\right)_{0 \leq n \leq N}$ of Problem (4.3) - (4.4) which belongs to this neighbourhood: For $1 \leq m \leq N$,

$$
\begin{aligned}
& \left\|\mathbf{u}-\mathbf{u}_{\tau}\right\|_{L^{\infty}\left(0, t_{m} ; L^{2}(\Omega)^{3}\right)}^{2}+\int_{0}^{t_{m}}\left\|\mathbf{u}(s)-\mathbf{u}_{\tau}(s)\right\|_{X(\Omega)}^{2} d s \\
& \quad \leq C\left(\sum_{n=1}^{m} \sum_{\kappa \in \mathcal{T}_{n h}}\left(\eta_{n, \kappa}^{\tau}\right)^{2}+\left\|\mathbf{f}-\pi_{\tau} \mathbf{f}\right\|_{L^{2}\left(0, t_{m} ; X(\Omega)^{\prime}\right)}^{2}+\sum_{n=1}^{m} \tau_{n}\left\|\mathbf{u}^{n}-\mathbf{u}_{h}^{n}\right\|_{X(\Omega)}^{2}\right) .
\end{aligned}
$$

Proof. Problem (2.3) can equivalently be written $\mathbf{u}-\mathcal{S} \mathbf{f}^{*}(\mathbf{u})=0$. Since the nonlinearity in (2.3) is quadratic, the mapping $D \mathbf{f}^{*}$ is obvuously Lipschitz-continuous with values in $L^{2}\left(0, T ; X(\Omega)^{\prime}\right)$ (this requires the assumption that $\Gamma_{m}$ does not contain any re-entrant corner). Thus applying [15, Theorem 1] (see also [17, Proposition 2.1]) yields

$$
\begin{array}{r}
\left\|\mathbf{u}-\mathbf{u}_{\tau}\right\|_{\left.L^{\infty}\left(0, t_{m} ; L^{2}(\Omega)^{3}\right)\right) \cap L^{2}\left(0, t_{m} ; X(\Omega)\right)} \leq c(\mathbf{u})\left\|\mathbf{u}_{\tau}-\mathcal{S} f^{*}\left(\mathbf{u}_{\tau}\right)\right\|_{\left.L^{\infty}\left(0, t_{m} L^{2}(\Omega)^{3}\right)\right) \cap L^{2}\left(0, t_{m} ; X(\Omega)\right)} \\
=c(\mathbf{u})\left\|\mathbf{u}-\mathbf{u}_{\tau}-\mathcal{S}\left(\mathbf{f}^{*}(\mathbf{u})-\mathbf{f}^{*}\left(\mathbf{u}_{\tau}\right)\right)\right\|_{L^{\infty}\left(0, T ; L^{2}(\Omega)^{3}\right) \cap L^{2}(0, T ; X(\Omega))} .
\end{array}
$$

Il follows from the properties of the operator $\mathcal{S}$ and the definition of $\mathbf{u}_{\tau}$ that this equation can equivalently be written

$$
\begin{aligned}
\left\|\mathbf{u}-\mathbf{u}_{\tau}\right\|_{L^{\infty}\left(0, t_{m} ; L^{2}(\Omega)^{3}\right)}^{2}+\int_{0}^{t_{m}}\left\|\mathbf{u}(s)-\mathbf{u}_{\tau}(s)\right\|_{X(\Omega)}^{2} d s & \\
& \leq c \int_{0}^{t_{m}}\left(\left\|R^{\tau}\left(\mathbf{u}_{\tau}\right)(s)\right\|_{X(\Omega)^{\prime}}^{2}+\left\|\left(\mathbf{f}-\pi_{\tau} \mathbf{f}\right)(s)\right\|_{X(\Omega)^{\prime}}^{2}\right) d s
\end{aligned}
$$

Thus using the first estimate in Lemma 4.3 and integrating with respect to time yields the desired estimate. 
To derive an a posteriori estimate between the solution $\mathbf{u}$ of Problem (2.3) and the solution $\mathbf{u}_{h}$ corresponding to the solutions $\mathbf{u}_{h}^{n}$ of (3.2)-(3.3), it suffices to obtain an a posteriori estimate between the solution $\mathbf{u}_{\tau}$ of Problem (4.3) - (4.4) and the solution $\mathbf{u}_{h}$, and to apply the triangle inequality using the previous theorem.

So, we now introduce the operator $\mathcal{S}_{\tau}$ which associates with any data $\mathbf{f}$ in $L^{2}\left(0, T ; X(\Omega)^{\prime}\right)$ the solution $u_{\tau}$ in $L^{\infty}\left(0, T ; L^{2}(\Omega)^{d}\right) \cap L^{2}(0, T ; X(\Omega))$ associated with the solution of Problem (4.3) - (4.4) in the linear case, i.e. when suppressing the term $\left(\operatorname{curl} \mathbf{u}^{n-1} \times \mathbf{u}^{n}, \mathbf{v}\right)$. We also set: $\mathbf{f}^{\tau}(\mathbf{u})=\pi_{\tau} \mathbf{f}-\operatorname{curl} \pi_{l, \tau} \mathbf{u} \times \pi_{\tau} \mathbf{u}$ and observe that problem (4.3) - (4.4) can equivalently be written $\mathbf{u}_{\tau}-\mathcal{S}_{\tau} \mathbf{f}^{\tau}\left(\mathbf{u}_{\tau}\right)=0$.

The proof of the next theorem is very similar to that of Theorem 4.4.

Theorem 4.5. Assume that the function $\mathbf{u}_{\tau}$ associated with the solution of Problem (4.3) - (4.4) is such that $\operatorname{Id}-\mathcal{S}_{\tau} D f^{\tau}(\mathbf{u})$ is an isomorphism of $L^{2}(0, T ; X(\Omega))$. When $\Omega$ has no re-entrant corner inside $\Gamma_{m}$, there exists a neighbourhood of $\mathbf{u}_{\tau}$ in $L^{2}(0, T ; X(\Omega))$ such that the following bound holds for any velocity $\mathbf{u}_{h}$ associated with the solutions $\left(\mathbf{u}_{h}^{n}\right)_{0 \leq n \leq N}$ of Problem (3.2) - (3.3) which belongs to this neighbourhood: For $1 \leq m \leq N$,

$$
\left\|\mathbf{u}_{\tau}-\mathbf{u}_{h}\right\|_{L^{\infty}\left(0, t_{m} ; L^{2}(\Omega)^{3}\right)}^{2}+\sum_{n=1}^{m} \tau_{n}\left\|\mathbf{u}^{n}-\mathbf{u}_{h}^{n}\right\|_{X(\Omega)}^{2} \leq c \sum_{n=1}^{m} \tau_{n}\left(\sum_{\kappa \in \mathcal{T}_{n h}} h_{\kappa}^{2}\left\|\mathbf{f}^{n}-\mathbf{f}_{h}^{n}\right\|_{0, \kappa}^{2}+\left(\eta_{n, \kappa}^{h}\right)^{2}\right) .
$$

Proof. We proceed in several steps.

1) For the same reasons as previously, the mapping $D f^{*}$ is obviously Lipschitz-continuous with values in $L^{2}\left(0, T ; X(\Omega)^{\prime}\right)$. Thus applying one more [15, Theorem 1] or [17, Proposition 2.1]) leads to

$$
\begin{aligned}
\left\|\mathbf{u}_{\tau}-\mathbf{u}_{h}\right\|_{L^{\infty}\left(0, t_{m} ; L^{2}(\Omega)^{3}\right)}^{2}+\int_{0}^{t_{m}} & \left\|\mathbf{u}_{\tau}(s)-\mathbf{u}_{h}(s)\right\|_{X(\Omega)}^{2} d s \leq c(\mathbf{u})\left\|\mathbf{u}_{h}-\mathcal{S}_{\tau} f^{\tau}\left(\mathbf{u}_{h}\right)\right\|_{L^{\infty}\left(0, t_{m} L^{2}(\Omega)^{3}\right) \cap L^{2}\left(0, t_{m} ; X(\Omega)\right.} \\
& =c(\mathbf{u})\left\|\mathbf{u}_{\tau}-\mathbf{u}_{h}-\mathcal{S}_{\tau}\left(f^{\tau}\left(\mathbf{u}_{\tau}\right)-f^{\tau}\left(\mathbf{u}_{h}\right)\right)\right\|_{L^{\infty}\left(0, T ; L^{2}(\Omega)^{3}\right) \cap L^{2}(0, T ; X(\Omega)) .}
\end{aligned}
$$

Il follows from the properties of the operator $\mathcal{S}_{\tau}$ and the definition of $\mathbf{u}_{h}$ that this equation can equivalently be written, see equation (4.10),

$$
\begin{aligned}
\left\|\mathbf{u}_{\tau}-\mathbf{u}_{h}\right\|_{L^{\infty}\left(0, t_{m} ; L^{2}(\Omega)^{3}\right)}^{2}+\int_{0}^{t_{m}}\left\|\mathbf{u}_{\tau}(s)-\mathbf{u}_{h}(s)\right\|_{X(\Omega)}^{2} & \\
& \leq c \int_{0}^{t_{m}} \sup _{\mathbf{v} \in X(\Omega)} \inf _{\mathbf{v}_{h} \in X_{h}} \frac{\left\langle\mathbf{f}^{n}-\mathbf{f}_{h}^{n}+R^{h}\left(\mathbf{u}_{h}\right), \mathbf{v}-\mathbf{v}_{\mathbf{h}}\right\rangle}{\|\mathbf{v}\|_{X(\Omega)}} d s .
\end{aligned}
$$

2) The fact that $\mathbf{u}_{\tau}-\mathbf{u}_{h}$ is piecewise affine, equal to $\mathbf{u}^{n}-\mathbf{u}_{h}^{n}$ at $t_{n}$, gives, by using Simpson formula,

$$
\begin{aligned}
& \int_{t_{n-1}}^{t_{n}}\left\|\mathbf{u}_{\tau}(s)-\mathbf{u}_{h}(s)\right\|_{X(\Omega)}^{2} d s=\frac{\tau_{n}}{3}\left(\left\|\mathbf{u}_{\tau}\left(t_{n}\right)-\mathbf{u}_{h}\left(t_{n}\right)\right\|_{X(\Omega)}^{2}+\left\|\mathbf{u}_{\tau}\left(t_{n-1}\right)-\mathbf{u}_{h}\left(t_{n-1}\right)\right\|_{X(\Omega)}^{2}\right. \\
& +\left(\operatorname{curl}\left(\mathbf{u}_{\tau}\left(t_{n-1}\right)-\mathbf{u}_{h}\left(t_{n-1}\right)\right), \operatorname{curl}\left(\mathbf{u}_{\tau}\left(t_{n}\right)-\mathbf{u}_{h}\left(t_{n}\right)\right)\right) \\
& \left.+\left(\operatorname{div}\left(\mathbf{u}_{\tau}\left(t_{n-1}\right)-\mathbf{u}_{h}\left(t_{n-1}\right)\right), \operatorname{div}\left(\mathbf{u}_{\tau}\left(t_{n}\right)-\mathbf{u}_{h}\left(t_{n}\right)\right)\right)\right)
\end{aligned}
$$

and the inequalites $a b \geq-\frac{1}{4} a^{2}-b^{2}$ and $a b \leq \frac{1}{2} a^{2}+\frac{1}{2} b^{2}$ yield the equivalence property

$$
\begin{aligned}
& \frac{\tau_{n}}{4}\left\|\mathbf{u}_{\tau}\left(t_{n}\right)-\mathbf{u}_{h}\left(t_{n}\right)\right\|_{X(\Omega)}^{2} \leq \int_{t_{n-1}}^{t_{n}}\left\|\mathbf{u}_{\tau}(s)-\mathbf{u}_{h}(s)\right\|_{X(\Omega)}^{2} d s \\
& \quad \leq \frac{\tau_{n}}{2}\left(\left\|\mathbf{u}_{\tau}\left(t_{n}\right)-\mathbf{u}_{h}\left(t_{n}\right)\right\|_{X(\Omega)}^{2}+\left\|\mathbf{u}_{\tau}\left(t_{n-1}\right)-\mathbf{u}_{h}\left(t_{n-1}\right)\right\|_{X(\Omega)}^{2}\right) .
\end{aligned}
$$

By using the relation $\tau_{n} \leq \sigma_{\tau} \tau_{n-1}$, the last inequality implies

$$
\frac{1}{4} \sum_{n=0}^{m} \tau_{n}\left\|\mathbf{u}^{n}-\mathbf{u}_{h}^{n}\right\|_{X(\Omega)}^{2} \leq \sum_{n=1}^{m} \int_{t_{n-1}}^{t_{n}}\left\|\mathbf{u}_{\tau}(s)-\mathbf{u}_{h}(s)\right\|_{X(\Omega)}^{2} d s \leq \frac{1+\sigma_{\tau}}{2} \sum_{n=0}^{m} \tau_{n}\left\|\mathbf{u}^{n}-\mathbf{u}_{h}^{n}\right\|_{X(\Omega)}^{2} .
$$

3) The term involving $R^{h}\left(\mathbf{u}_{h}\right)$ is evaluated in the second estimate in Lemma 4.3 and a similar argument leads to estimate the term concerning $\mathbf{f}^{n}-\mathbf{f}_{h}^{n}$. 
Remark 4.6. When the convergence of $\mathcal{S}_{\tau}$ to $\mathcal{S}$ when $|\tau|$ tends to zero is established, it is readily checked that the assumption " $\mathrm{Id}-\mathcal{S} D f^{*}(\mathbf{u})$ is an isomorphism of $L^{2}(0, T ; X(\Omega))$ " implies its discrete analogue, namely " $\mathrm{Id}-\mathcal{S}_{\tau} D f^{\tau}(\mathbf{u})$ is an isomorphism of $L^{2}(0, T ; X(\Omega))$ ", at least for $|\tau|$ small enough

Remark 4.7. When $\Omega$ has at least a re-entrant corner inside $\Gamma_{m}$, it is only known [10] that $X(\Omega)$ is contained in $H^{\frac{1}{2}}(\Omega)$. So the previous estimate has to be replaced by

$$
\left\|\mathbf{u}_{\tau}-\mathbf{u}_{h}\right\|_{L^{\infty}\left(0, t_{m} ; L^{2}(\Omega)^{2}\right)}^{2}+\sum_{n=1}^{m} \tau_{n}\left\|\mathbf{u}^{n}-\mathbf{u}_{h}^{n}\right\|_{X(\Omega)}^{2} \leq c \sum_{n=1}^{m} \tau_{n}\left(\sum_{\kappa \in \mathcal{T}_{n h}} h_{\kappa}\left\|\mathbf{f}^{n}-\mathbf{f}_{h}^{n}\right\|_{0, \kappa}^{2}+h_{\kappa}^{-1}\left(\eta_{n, \kappa}^{h}\right)^{2}\right) .
$$

Thus a lack of optimality of $\max _{\kappa \in \mathcal{T}_{n h}} h_{\kappa}^{-\frac{1}{2}}$ occurs in the upper bound.

Corollary 4.8. If the assumptions of Theorems 4.4 and 4.5 are satisfied, the following a posteriori error estimate holds between the velocity $\mathbf{u}$ solution of Problem (2.3) and the velocity $\mathbf{u}_{h}$ corresponding to the solutions $\mathbf{u}_{h}^{n}$ of Problem (3.2) - (3.3)

$$
\begin{aligned}
& \left\|\mathbf{u}-\mathbf{u}_{h}\right\|_{L^{\infty}\left(0, t_{m} ; L^{2}(\Omega)^{3}\right)}^{2}+\int_{0}^{t_{m}}\left\|\mathbf{u}(s)-\mathbf{u}_{h}(s)\right\|_{X(\Omega)}^{2} d s \\
& \quad \leq C\left(\sum_{n=1}^{m} \sum_{\kappa \in \mathcal{T}_{n h}}\left(\tau_{n}\left(\eta_{n, \kappa}^{h}\right)^{2}+\left(\eta_{n, \kappa}^{\tau}\right)^{2}+\tau_{n} h_{\kappa}^{2}\left\|\mathbf{f}^{n}-\mathbf{f}_{h}^{n}\right\|_{0, \kappa}^{2}\right)+\left\|\mathbf{f}-\pi_{\tau} \mathbf{f}\right\|_{L^{2}\left(0, t_{m} ; X(\Omega)^{\prime}\right)}^{2} .\right.
\end{aligned}
$$

Proof. The proof is a direct consequence of Theorems 4.4 and 4.5 , combined with the triangle inequality

$$
\begin{aligned}
& \left\|\mathbf{u}-\mathbf{u}_{h}\right\|_{L^{\infty}\left(0, t_{n} ; L^{2}(\Omega)^{3}\right)}^{2}+\int_{0}^{t_{n}}\left\|\mathbf{u}(s)-\mathbf{u}_{h}(s)\right\|_{X(\Omega)}^{2} d s \leq 2\left\|\mathbf{u}-\mathbf{u}_{\tau}\right\|_{L^{\infty}\left(0, t_{n} ; L^{2}(\Omega)^{2}\right)}^{2} \\
& +2 \int_{0}^{t_{n}}\left\|\mathbf{u}(s)-\mathbf{u}_{\tau}(s)\right\|_{X(\Omega)}^{2} d s+2\left\|\mathbf{u}_{\tau}-\mathbf{u}_{h}\right\|_{L^{\infty}\left(0, t_{n} ; L^{2}(\Omega)^{2}\right)}^{2}+2 \int_{0}^{t_{n}}\left\|\mathbf{u}_{\tau}(s)-\mathbf{u}_{h}(s)\right\|_{X(\Omega)}^{2} d s .
\end{aligned}
$$

and the equivalence property (4.19).

Next, we bound the function $\frac{\partial}{\partial t}\left(\mathbf{u}-\mathbf{u}_{h}\right)+\left(\operatorname{curl} \mathbf{u} \times \mathbf{u}-\operatorname{curl} \pi_{l, \tau} \mathbf{u}_{h} \times \pi_{\tau} \mathbf{u}_{h}\right)+\nabla\left(p-\pi_{\tau} p_{h}\right)$.

Theorem 4.9. If the domain $\Omega$ has no re-entrant corner inside $\Gamma_{m}$, the following a posteriori error estimate holds between the solution $(\mathbf{u}, p)$ of problem (2.3) and the pair $\left(\mathbf{u}_{h}, \pi_{\tau} p_{h}\right)$ associated with the solutions of Problem (3.2) - (3.3): For $1 \leq m \leq N$,

$$
\begin{aligned}
\| \frac{\partial}{\partial t}(\mathbf{u} & \left.-\mathbf{u}_{h}\right)+\left(\mathbf{c u r l} \mathbf{u} \times \mathbf{u}-\mathbf{c u r l} \pi_{l, \tau} \mathbf{u}_{h} \times \pi_{\tau} \mathbf{u}_{h}\right)+\nabla\left(p-\pi_{\tau} p_{h}\right) \|_{L^{2}\left(0, t_{m} ; X(\Omega)^{\prime}\right)} \\
& \leq C\left(\sum_{n=1}^{m} \sum_{\kappa \in \mathcal{T}_{n h}}\left(\tau_{n}\left(\eta_{n, \kappa}^{h}\right)^{2}+\left(\eta_{n, \kappa}^{\tau}\right)^{2}\right)+\sum_{n=0}^{m} \tau_{n} \sum_{\kappa \in \mathcal{T}_{n h}} h_{\kappa}^{2}\left\|\mathbf{f}^{n}-\mathbf{f}_{h}^{n}\right\|_{0, \kappa}^{2}+\left\|\mathbf{f}-\pi_{\tau} \mathbf{f}\right\|_{L^{2}\left(0, t_{m} ; X(\Omega)^{\prime}\right)}^{2}\right) .
\end{aligned}
$$

Proof. By subtracting (3.2) - (3.3) from (2.3), we derive

$$
\begin{aligned}
\| \frac{\partial}{\partial t}(\mathbf{u} & \left.-\mathbf{u}_{h}\right)(t)+\left(\operatorname{curl} \mathbf{u}(t) \times \mathbf{u}(t)-\operatorname{curl} \pi_{l, \tau} \mathbf{u}_{h}(t) \times \pi_{\tau} \mathbf{u}_{h}(t)\right)+\nabla\left(p(t)-\pi_{\tau} p_{h}(t)\right) \|_{X(\Omega)^{\prime}} \\
& =\sup _{\mathbf{v} \in X(\Omega)} \frac{-\nu\left(\operatorname{curl}\left(\mathbf{u}(t)-\mathbf{u}_{h}(t)\right), \mathbf{c u r l} \mathbf{v}\right)-\nu\left(\operatorname{div}\left(\mathbf{u}(t)-\mathbf{u}_{h}(t)\right), \operatorname{div} \mathbf{v}\right)+\left\langle R\left(\mathbf{u}_{h}\right)(t), \mathbf{v}\right\rangle}{\|\mathbf{v}\|_{X(\Omega)}},
\end{aligned}
$$

where the residual $R\left(\mathbf{u}_{h}\right)(t)$ is given by, for $t$ in $\left.] t_{n-1}, t_{n}\right]$;

$$
R\left(\mathbf{u}_{h}\right)=\left(\mathbf{f}-\mathbf{f}^{n}\right)+\left(\mathbf{f}^{n}-\mathbf{f}_{h}^{n}\right)+R^{h}\left(\mathbf{u}_{h}\right)+R^{\tau}\left(\mathbf{u}_{h}\right),
$$

For the first term in the right-hand side, we have

$$
\nu\left(\operatorname{curl}\left(\mathbf{u}(t)-\mathbf{u}_{h}(t)\right), \operatorname{curl} \mathbf{v}\right)+\nu\left(\operatorname{div}\left(\mathbf{u}(t)-\mathbf{u}_{h}(t)\right), \operatorname{div} \mathbf{v}\right) \leq \nu\left\|\mathbf{u}(t)-\mathbf{u}_{h}(t)\right\|\left\|_{X(\Omega)}\right\| \mathbf{v} \|_{X(\Omega)} .
$$


We use Lemma 4.3 to bound the second term of the right-hand side. Finally, by integrating over $t$ from $t_{n-1}$ to $t_{n}$, summing over $n$ and using Corollary 4.8, we obtain the result.

To conclude the upper bound, we estimate the quantity $\sum_{n=1}^{m} \int_{t_{m-1}}^{t_{m}}\left\|\mathbf{u}(t)-\pi_{\tau} \mathbf{u}_{h}(t)\right\|_{X(\Omega)}^{2} d t$.

Theorem 4.10. The following a posteriori error estimate holds between the velocity $\mathbf{u}$ solution of Problem (2.3) and the velocity $\mathbf{u}_{h}$ corresponding to the solutions $\mathbf{u}_{h}^{n}$ of Problem (3.2) - (3.3)

$$
\sum_{n=1}^{m} \int_{t_{n-1}}^{t_{n}}\left\|\mathbf{u}(s)-\pi_{\tau} \mathbf{u}_{h}(s)\right\|_{X(\Omega)}^{2} d s \leq C\left(\int_{0}^{t_{n}}\left\|\mathbf{u}(s)-\mathbf{u}_{h}(s)\right\|_{X(\Omega)}^{2} d s+\sum_{n=1}^{m} \sum_{\kappa \in \mathcal{T}_{n h}}\left(\eta_{n, \kappa}^{\tau}\right)^{2}\right) .
$$

Proof. We consider the velocity $\mathbf{u}$ solution of Problem (2.3) and the velocity $\mathbf{u}_{h}$ corresponding to the solutions $\mathbf{u}_{h}^{n}$ of Problem (3.2) - (3.3). We have for $t$ in $\left.] t_{n-1}, t_{n}\right]$ :

$$
\begin{aligned}
\left\|\mathbf{u}(t)-\pi_{\tau} \mathbf{u}_{h}(t)\right\|_{X(\Omega)}^{2} & \leq\left(\left\|\mathbf{u}(t)-\mathbf{u}_{h}(t)\right\|_{X(\Omega)}+\left\|\mathbf{u}_{h}(t)-\mathbf{u}_{h}^{n}\right\|_{X(\Omega)}\right)^{2} \\
& \leq\left(\left\|\mathbf{u}(t)-\mathbf{u}_{h}(t)\right\|_{X(\Omega)}+\frac{t-t_{n}}{\tau_{n}}\left\|\mathbf{u}_{h}^{n}-\mathbf{u}_{h}^{n-1}\right\|_{X(\Omega)}\right)^{2} \\
& \leq 2\left(\left\|\mathbf{u}(t)-\mathbf{u}_{h}(t)\right\|_{X(\Omega)}^{2}+\left(\frac{t-t_{n}}{\tau_{n}}\right)^{2}\left\|\mathbf{u}_{h}^{n}-\mathbf{u}_{h}^{n-1}\right\|_{X(\Omega)}^{2}\right) .
\end{aligned}
$$

When integrating between $t_{n-1}$ and $t_{n}$ and summing over $n$, the estimate follows from the definition of the $\eta_{n, \kappa}^{\tau}$.

The bounds (4.20), (4.22) and (4.25) constitute our upper bounds.

4.3. Upper bounds of the indicators. We now prove upper bounds of the indicators (or equivalently lower bounds of the error) and we begin with the term $\eta_{n, \kappa}^{h}$.

Theorem 4.11. Each indicator $\eta_{n, \kappa}^{h}, \kappa \in \mathcal{T}_{n h}$, defined in (4.14) satisfy the following upper bound

$$
\begin{aligned}
& \tau_{n}\left(\eta_{n, \kappa}^{h}\right)^{2} \leq \\
& c\left(\left\|\frac{\partial}{\partial t}\left(\mathbf{u}-\mathbf{u}_{h}\right)(t)+\left(\mathbf{c u r l} \mathbf{u}(t) \times \mathbf{u}(t)-\operatorname{curl} \pi_{t, \tau} u_{h}(t) \times \pi_{\tau} \mathbf{u}_{h}(t)\right)+\nabla\left(p(t)-\pi_{\tau} p_{h}(t)\right)\right\|_{L^{2}\left(t_{n-1}, t_{n} ; X\left(\omega_{\kappa}\right)^{\prime}\right)}^{2}\right. \\
& \left.+\nu\left\|\mathbf{u}-\mathbf{u}_{h}^{n}\right\|_{L^{2}\left(t_{n-1}, t_{n} ; X\left(\omega_{\kappa}\right)\right)}^{2}+\left\|\mathbf{f}-\mathbf{f}^{n}\right\|_{L^{2}\left(t_{n-1}, t_{n} ; X\left(\omega_{\kappa}\right)^{\prime}\right)}^{2}+\tau_{n} h_{\kappa}^{2}\left\|\mathbf{f}^{n}-\mathbf{f}_{h}^{n}\right\|_{0, \omega_{\kappa}}^{2}\right),
\end{aligned}
$$

where $\omega_{\kappa}$ denotes the union of the elements of $\mathcal{T}_{n h}$ that share at least a face with $\kappa$.

Proof. We denote by $L(W)$ the right-hand side of (4.26) for the domain $\omega_{\kappa}$ replaced by a domain $W$. The solution $\mathbf{u}$ of Problem (2.3) and the solution velocity $\mathbf{u}_{h}$ associated with the solution $\left(\mathbf{u}_{h}^{n}\right)_{0 \leq n \leq N}$ of Problem $(3.2)-(3.3)$ verify : For all $\mathbf{v}$ in $X(\Omega), \mathbf{v}_{h}$ in $X_{n h}$ and $t$ in $\left.] t_{n-1}, t_{n}\right](1 \leq n \leq N)$,

$$
\begin{aligned}
& \left(\frac{\partial}{\partial t}\left(\mathbf{u}-\mathbf{u}_{h}\right)(t), \mathbf{v}(t)\right)+\nu\left(\operatorname{curl}\left(\mathbf{u}(t)-\mathbf{u}_{h}^{n}\right), \operatorname{curl} \mathbf{v}(t)\right)+\nu\left(\operatorname{div}\left(\mathbf{u}(t)-\mathbf{u}_{h}^{n}\right), \operatorname{div} \mathbf{v}(t)\right) \\
& +\left(\operatorname{curl} \mathbf{u}(t) \times \mathbf{u}(t)-\operatorname{curl} \pi_{l, \tau} \mathbf{u}_{h}(t) \times \pi_{\tau} \mathbf{u}_{h}(t)\right)-\left(\operatorname{div} \mathbf{v}(t),\left(p(t)-\pi_{\tau} p_{h}(t)\right)\right) \\
& =\left\langle\mathbf{f}(t)-\mathbf{f}^{n}, \mathbf{v}(t)\right\rangle+\left\langle\mathbf{f}^{n}-\mathbf{f}_{h}^{n}+R^{h}\left(\mathbf{u}_{h}\right), \mathbf{v}(t)-\mathbf{v}_{h}(t)\right\rangle .
\end{aligned}
$$

Next, we estimate successively every term of $\eta_{n, \kappa}^{h}$.

1) First of all, we take $\mathbf{v}_{h}=\mathbf{0}$ and

$\mathbf{v}=\mathbf{v}_{\kappa}= \begin{cases}\left(\mathbf{f}_{h}^{n}-\frac{1}{\tau_{n}}\left(\mathbf{u}_{h}^{n}-\mathbf{u}_{h}^{n-1}\right)-\operatorname{curl} \mathbf{u}_{n}^{n-1} \times \mathbf{u}_{h}^{n}-\nabla p_{h}^{n}-\nu \operatorname{curl} \operatorname{curl} \mathbf{u}_{h}^{n}+\nu \nabla \operatorname{div} \mathbf{u}_{h}^{n}\right) \psi_{\kappa} & \text { on } \kappa \\ 0 & \text { on } \Omega \backslash \kappa\end{cases}$ 
and we integrate between $t_{n-1}$ and $t_{n}$ to obtain:

$$
\begin{aligned}
& \tau_{n}\left\|\left(\mathbf{f}_{h}^{n}-\frac{1}{\tau_{n}}\left(\mathbf{u}_{h}^{n}-\mathbf{u}_{h}^{n-1}\right)-\mathbf{c u r l} \mathbf{u}_{n}^{n-1} \times \mathbf{u}_{h}^{n}-\nabla p_{h}^{n}-\nu \operatorname{curl} \mathbf{c u r l} \mathbf{u}_{h}^{n}+\nu \nabla \operatorname{div} \mathbf{u}_{h}^{n}\right) \psi_{\kappa}^{1 / 2}\right\|_{L^{2}(\kappa)^{d}}^{2} \\
& \leq c\left(\tau_{n}^{1 / 2} \| \frac{\partial}{\partial t}\left(\mathbf{u}-\mathbf{u}_{h}\right)(t)+\left(\operatorname{curl} \mathbf{u}(t) \times \mathbf{u}(t)-\operatorname{curl} \pi_{t, \tau} \mathbf{u}_{h}(t) \times \pi_{\tau} \mathbf{u}_{h}(t)\right)\right. \\
& \quad+\nabla\left(p(t)-\pi_{\tau} p_{\tau}(t)\right)||_{L^{2}\left(t_{n-1}, t_{n} ; X(\kappa)^{\prime}\right)}\left|\mathbf{v}_{\kappa}\right|_{1, \kappa} \\
& \quad+\nu \tau_{n}^{1 / 2}\left\|\mathbf{u}-\mathbf{u}_{h}^{n}\right\|_{L^{2}\left(t_{n-1}, t_{n} ; X(\kappa)\right)}\left|\mathbf{v}_{\kappa}\right|_{1, \kappa}+\tau_{n}^{1 / 2}\left\|\mathbf{f}-\mathbf{f}^{n}\right\|_{L^{2}\left(t_{n-1}, t_{n} ; X(\kappa)^{\prime}\right)}\left\|_{\mathbf{v}_{\kappa}}\right\|_{X(\kappa)} \\
& \left.\quad+\tau_{n}\left\|\mathbf{f}^{n}-\mathbf{f}_{h}^{n}\right\|_{0, \kappa}\left\|\mathbf{v}_{\kappa}\right\|_{0, \kappa}\right)
\end{aligned}
$$

By using the inequality $a b \leq 2 a^{2}+\frac{1}{8} b^{2}$ for all the terms of the right-hand side, multiplying by $h_{\kappa}^{2}$, remarking that $\left\|\mathbf{v}_{\kappa}\right\|_{X(\kappa)} \leq c\left|\mathbf{v}_{\kappa}\right|_{1, \kappa}$ and thanks to Property 4.1, we obtain

$$
\tau_{n} h_{\kappa}^{2}\left\|\left(\mathbf{f}_{h}^{n}-\frac{1}{\tau_{n}}\left(\mathbf{u}_{h}^{n}-\mathbf{u}_{h}^{n-1}\right)-\operatorname{curl} \mathbf{u}_{n}^{n-1} \times \mathbf{u}_{h}^{n}-\nabla p_{h}^{n}-\nu \operatorname{curl} \operatorname{curl} \mathbf{u}_{h}^{n}+\nu \nabla \operatorname{div} \mathbf{u}_{h}^{n}\right) \psi_{\kappa}^{1 / 2}\right\|_{0, \kappa}^{2} \leq L(\kappa) .
$$

2) Second, we take $\mathbf{v}_{h}=\mathbf{0}$ and for any $e \in \varepsilon_{\kappa}$, we denote by $\kappa^{\prime}$ the other element containing $e$. We introduce the function

$$
R_{n, e}^{h}=\left\{\begin{array}{l}
{\left[\operatorname{curl} \mathbf{u}_{h}^{n} \times \mathbf{n}+\nu\left(\operatorname{div} \mathbf{u}_{h}^{n}\right) \mathbf{n}-p_{h}^{n} \mathbf{n}\right]_{e}} \\
\operatorname{curl} \mathbf{u}_{h}^{n} \times \mathbf{n} \quad \text { if } e \in \varepsilon_{\kappa}^{m}
\end{array}\right.
$$

and we take $\mathbf{v}=\mathbf{v}_{e}=\mathcal{L}_{e}\left(R_{n, e}^{h} \psi_{e}\right)$ extended by zero to $\Omega$. Then, we integrate between $t_{n-1}$ and $t_{n}$ to obtain:

$$
\begin{aligned}
& \tau_{n}\left\|R_{n, e}^{h} \psi_{e}^{1 / 2}\right\|_{L^{2}(e)^{d}}^{2} \leq \\
& c\left(\tau_{n}\left\|\left(\mathbf{f}_{h}^{n}-\frac{1}{\tau_{n}}\left(\mathbf{u}_{h}^{n}-\mathbf{u}_{h}^{n-1}\right)-\operatorname{curl} \mathbf{u}_{n}^{n-1} \times \mathbf{u}_{h}^{n}-\nabla p_{h}^{n}-\nu \operatorname{curl} \operatorname{curl} \mathbf{u}_{h}^{n}+\nu \nabla \operatorname{div} \mathbf{u}_{h}^{n}\right)\right\|_{\left.0, \kappa \cup \kappa^{\prime}\right)}\left\|\mathbf{v}_{e}\right\|_{0, \kappa \cup \kappa^{\prime}}\right. \\
& +\tau_{n}^{1 / 2} \| \frac{\partial}{\partial t}\left(\mathbf{u}-\mathbf{u}_{h}\right)(t) \\
& -\left(\operatorname{curl} \mathbf{u}(t) \times \mathbf{u}(t)-\operatorname{curl} \pi_{i, \tau} \mathbf{u}_{h}(t) \times \pi_{\tau} \mathbf{u}_{h}(t)\right)-\nabla\left(p(t)-\pi_{\tau} p_{\tau}(t)\right)||_{L^{2}\left(t_{n-1}, t_{n} ; X\left(\kappa \cup \kappa^{\prime}\right)^{\prime}\right)}\left|\mathbf{v}_{e}\right|_{1, \kappa \cup \kappa^{\prime}} \\
& +\nu \tau_{n}^{1 / 2}\left\|\mathbf{u}-\mathbf{u}_{h}^{n}\right\|_{L^{2}\left(t_{n-1}, t_{n} ; X\left(\kappa \cup \kappa^{\prime}\right)\right)}\left|\mathbf{v}_{e}\right|_{1, \kappa \cup \kappa^{\prime}}+\tau_{n}^{1 / 2}\left\|\mathbf{f}-\mathbf{f}^{n}\right\|_{L^{2}\left(t_{n-1}, t_{n} ; X\left(\kappa \cup \kappa^{\prime}\right)^{\prime}\right)}\left\|\mathbf{v}_{e}\right\|_{X\left(\kappa \cup \kappa^{\prime}\right)} \\
& \left.+\tau_{n}\left\|\mathbf{f}^{n}-\mathbf{f}_{h}^{n}\right\|_{0, \kappa \cup \kappa^{\prime}}\left\|\mathbf{v}_{e}\right\|_{0, \kappa \cup \kappa^{\prime}}\right) \text {. }
\end{aligned}
$$

By using the inequality $a b \leq 2 a^{2}+\frac{1}{8} b^{2}$ for all the terms of the right-hand side, multiplying by $h_{e}$, remarking that || $\mathbf{v}_{\kappa} \|_{X(\kappa)} \leq c\left|\mathbf{v}_{\kappa}\right|_{1, \kappa}$, using Property 4.1 and summing over $\partial \kappa$, we obtain:

$$
\tau_{n}\left(\sum_{e \in \varepsilon_{\kappa}} h_{e}\left\|\mathbf{c u r l} \mathbf{u}_{h}^{n} \times \mathbf{n}+\nu\left(\operatorname{div} \mathbf{u}_{h}^{n}\right) \mathbf{n}-p_{h}^{n} \mathbf{n}\right\|_{0, e}^{2}+\sum_{e \in \varepsilon_{\kappa}^{m}} h_{e}\left\|\operatorname{curl} \mathbf{u}_{h}^{n} \times \mathbf{n}\right\|_{0, e}^{2}\right) \leq L\left(\omega_{\kappa}\right)
$$

3) Finally, we take in the equation (4.11)

$$
q(t)=q_{\kappa}(t)=\operatorname{div} \mathbf{u}_{h}(t) \xi_{\kappa},
$$

where $\xi_{\kappa}$ denotes the characteristic function of $\kappa$, and we integrate between $t_{n-1}$ and $t_{n}$ to obtain

$$
\left\|\operatorname{div} \mathbf{u}_{h}\right\|_{L^{2}\left(t_{n-1}, t_{n} ; L^{2}(\kappa)\right)} \leq\left\|\operatorname{div}\left(\mathbf{u}-\mathbf{u}_{h}\right)\right\|_{L^{2}\left(t_{n-1}, t_{n} ; L^{2}(\kappa)\right)} .
$$

The same argument which gives the inequality (4.5), can be applied here and gives

$$
\begin{aligned}
\frac{1}{4} \tau_{n}\left\|\operatorname{div} \mathbf{u}_{h}^{n}\right\|_{0, \kappa} & \leq\left\|\operatorname{div} \mathbf{u}_{h}\right\|_{L^{2}\left(t_{n-1}, t_{n} ; L^{2}(\kappa)\right)} \\
& \leq\left\|\mathbf{u}-\mathbf{u}_{h}\right\|_{L^{2}\left(t_{n-1}, t_{n} ; X(\kappa)\right)} .
\end{aligned}
$$

Combining estimates (4.28), (4.31) and (4.32) leads to desired results.

To finish the lower bound, we have to estimate the term $\eta_{n, \kappa}^{\tau}$. 
Theorem 4.12. Each indicator $\eta_{n, \kappa}^{\tau}, \kappa \in \mathcal{T}_{n h}$, defined in (4.13) satisfy the following upper bound

$$
\begin{aligned}
& \left(\eta_{n, \kappa}^{\tau}\right)^{2} \leq \\
& c\left(\left\|\frac{\partial}{\partial t}\left(\mathbf{u}-\mathbf{u}_{h}\right)(t)+\left(\mathbf{c u r l} \mathbf{u}(t) \times \mathbf{u}(t)-\mathbf{c u r l} \pi_{l, \tau} \mathbf{u}_{h}(t) \times \pi_{\tau} \mathbf{u}(t)\right)+\nabla\left(p(t)-\pi_{\tau} p_{\tau}(t)\right)\right\|_{L^{2}\left(t_{n-1}, t_{n} ; X(\kappa)^{\prime}\right)}^{2}\right. \\
& \left.+\left\|\mathbf{u}-\mathbf{u}_{h}\right\|_{L^{2}\left(t_{n-1}, t_{n} ; X(\kappa)\right)}^{2}+\left\|\mathbf{f}-\mathbf{f}^{n}\right\|_{L^{2}\left(t_{n-1}, t_{n} ; X(\kappa)^{\prime}\right)}^{2}+\tau_{n} h_{\kappa}^{2}\left\|\mathbf{f}^{n}-\mathbf{f}_{h}^{n}\right\|_{0, \kappa}^{2}+\tau_{n}\left(\eta_{n, \kappa}^{h}\right)^{2}\right) .
\end{aligned}
$$

Proof. We sum up Equations (4.6) and (4.10). Owing to the definition of the operators $R^{h}$ and $R^{\tau}$, we obtain : For all $\mathbf{v} \in X(\Omega), \mathbf{v}_{h} \in X_{n h}$ and $\left.\left.t \in\right] t_{n-1}, t_{n}\right](1 \leq n \leq N)$,

$$
\begin{gathered}
\left(\frac{\partial}{\partial t}\left(\mathbf{u}-\mathbf{u}_{h}\right)(t), \mathbf{v}(t)\right)+\nu\left(\operatorname{curl}\left(\mathbf{u}(t)-\mathbf{u}_{h}(t)\right), \operatorname{curl} \mathbf{v}(t)\right)+\nu\left(\operatorname{div}\left(\mathbf{u}(t)-\mathbf{u}_{h}(t)\right), \operatorname{div} \mathbf{v}(t)\right) \\
+\left(\operatorname{curl} \mathbf{u}(t) \times \mathbf{u}(t)-\operatorname{curl} \pi_{l, \tau} \mathbf{u}_{h}(t) \times \pi_{\tau} \mathbf{u}(t)\right)-\left(\operatorname{div} \mathbf{v}(t),\left(p(t)-\pi_{\tau} p_{h}(t)\right)\right)=\left\langle\left(\mathbf{f}(t)-\mathbf{f}^{n}\right), \mathbf{v}(t)\right\rangle \\
+\left\langle\left(\mathbf{f}^{n}-\mathbf{f}_{h}^{n}\right)+R^{h}\left(\mathbf{u}_{h}\right), \mathbf{v}(t)-\mathbf{v}_{h}(t)\right\rangle+\left\langle\left(R^{\tau}\left(\mathbf{u}_{h}\right), \mathbf{v}(t)\right\rangle .\right.
\end{gathered}
$$

By taking

$$
\mathbf{v}=\mathbf{v}_{\kappa}= \begin{cases}\left(\mathbf{u}_{h}^{n}-\mathbf{u}_{h}^{n-1}\right) \psi_{\kappa} & \text { on } \kappa \\ 0 & \text { on } \Omega \backslash \kappa,\end{cases}
$$

and $\mathbf{v}_{h}=\mathcal{C}_{n h} \mathbf{v}$, and by integrating between $t_{n-1}$ and $t_{n}$ and by using the Cauchy-Schwarz inequality, we obtain:

$$
\begin{aligned}
\nu \frac{1}{2} \tau_{n} \| & \left(\mathbf{u}_{h}^{n}-\mathbf{u}_{h}^{n-1}\right) \psi_{\kappa}^{1 / 2} \|_{X(\kappa)}^{2} \\
\leq & c\left(\tau_{n}^{1 / 2} \| \frac{\partial}{\partial t}\left(\mathbf{u}-\mathbf{u}_{h}\right)(t)+\left(\mathbf{c u r l} \mathbf{u}(t) \times \mathbf{u}(t)-\operatorname{curl} \pi_{l, \tau} \mathbf{u}_{h}(t) \times \pi_{\tau} \mathbf{u}_{h}(t)\right)\right. \\
& \quad+\nabla\left(p(t)-\pi_{\tau} p_{\tau}(t)\right) \|_{L^{2}\left(t_{n-1}, t_{n} ; X(\kappa)^{\prime}\right)}\left|\mathbf{v}_{\kappa}\right|_{1, \kappa} \\
& +\nu \tau_{n}^{1 / 2}|| \mathbf{u}-\mathbf{u}_{h} \|_{L^{2}\left(t_{n-1}, t_{n} ; X(\kappa)\right)}\left|\mathbf{v}_{\kappa}\right|_{1, \kappa} \\
& +\tau_{n}^{1 / 2}|| \mathbf{f}-\mathbf{f}^{n} \|_{L^{2}\left(t_{n-1}, t_{n} ; X(\kappa)^{\prime}\right)}\left|\mathbf{v}_{\kappa}\right|_{X(\kappa)} \\
& \left.+\tau_{n} h_{\kappa}|| \mathbf{f}^{n}-\left.\mathbf{f}_{h}^{n}\right|_{0, \kappa}\left|\mathbf{v}_{\kappa}\right|_{1, \kappa}+\tau_{n} \gamma_{n, \kappa}\left|\mathbf{v}_{\kappa}\right|_{1, \kappa}\right)
\end{aligned}
$$

By using the inequality $a b \leq 2 a^{2}+\frac{1}{8} b^{2}$ for all the right hand side and Property 4.1, we obtain the result.

4.4. Conclusions. We have proved that, when the domain $\Omega$ has no re-entrant corner inside $\Gamma_{m}$, the pressure and the velocity verify the upper bound:

$$
\begin{aligned}
& \left\|\mathbf{u}-\mathbf{u}_{h}\right\|_{L^{\infty}\left(0, t_{m} ; L^{2}(\Omega)^{3}\right)}^{2}+\int_{0}^{t_{m}}\left\|\mathbf{u}(s)-\mathbf{u}_{h}(s)\right\|_{X(\Omega)}^{2}+\int_{0}^{t_{m}}\left\|\mathbf{u}(s)-\pi_{\tau} \mathbf{u}_{h}(s)\right\|_{X(\Omega)}^{2} d s \\
& +\left\|\frac{\partial}{\partial t}\left(\mathbf{u}-\mathbf{u}_{h}\right)(t)+\left(\mathbf{c u r l} \mathbf{u}(t) \times \mathbf{u}(t)-\mathbf{c u r l} \pi_{l, \tau} \mathbf{u}_{h}(t) \times \pi_{\tau} \mathbf{u}_{h}(t)\right)+\nabla\left(p(t)-\pi_{\tau} p_{h}(t)\right)\right\|_{L^{2}\left(0, t_{m} ; X(\Omega)^{\prime}\right)}^{2} \\
& \quad \leq C\left(\sum_{n=1}^{m} \sum_{\kappa \in \mathcal{T}_{n h}}\left(\tau_{n}\left(\eta_{n, \kappa}^{h}\right)^{2}+\left(\eta_{n, \kappa}^{\tau}\right)^{2}\right)+\sum_{n=1}^{m} \tau_{n} \sum_{\kappa \in \mathcal{T}_{n h}} h_{\kappa}^{2}\left\|\mathbf{f}^{n}-\mathbf{f}_{h}^{n}\right\|_{0, \kappa}^{2}+\left\|\mathbf{f}-\pi_{\tau} \mathbf{f}\right\|_{L^{2}\left(0, t_{m} ; X(\Omega)^{\prime}\right)}^{2}\right),
\end{aligned}
$$

while the lower bounds follow from (4.33) and (4.26).

We observe that estimate (4.35) is optimal: Up to the terms involving the data, the full error is bounded from above and from below by a constant times the sum of all indicators. Estimates (4.33) and (4.26) are local in space and local in time. The indicators $\eta_{n, \kappa}^{\tau}$ can be interpreted as a measure for the error of the time discretization. Correspondingly, they can be used for controlling the step-size in time. On the other hand, the other indicators $\eta_{n, \kappa}^{h}$ can be viewed as a measure for the error of the space discretization and can be used to adapt the mesh size in space. We refer to [7, Section 6] for the detailed description of a simple adaptivity strategy relying on similar estimates. 


\section{NumericAl RESUlts}

To validate the theoretical results, we performed several numerical simulations using the FreeFem ++ software (see [14]). We consider the two-dimensional square $\Omega=] 0,3[\times] 0,3\left[\right.$ with $\Gamma_{m}=[0,3] \times\{0\} \cup\{0\} \times$ $[0,3]$ and $\Gamma=[0,3] \times\{3\} \cup\{3\} \times[0,3]$, and we choose $\nu=1$. The numerical velocity and the pressure are taken as $(\mathbf{u}, p)=(\operatorname{curl} \psi, p)$,

$$
\begin{aligned}
& \psi(x, y, t))=t e^{-\alpha(y-y 0-\beta t)^{2}-\alpha(x-x 0-\beta t)^{2}}, \\
& \text { and } p(x, y, t)=e^{-t} \cos (2 / 3 \pi x) \sin (2 / 3 \pi y),
\end{aligned}
$$

where $\alpha=50, \beta=1$ and $\left(x_{0}, y_{0}\right)=(1,1)$. The velocity is a Gaussian which is null for $t=0$ and which is contained in a small ball and moving on the diagonal of $\Omega$. The numerical experiments are done between $t=0$ and $t=1.25$ to be sure that the ball do not touch the border $\Gamma$. Our goal is to validate the a posteriori estimates by mesh adaptation: we show that the corresponding mesh follow the numerical solution.

We start the numerical test with the initial time step $d t=1 / 20$ and with the following initial mesh (FIGURE 1). The figures (FIGURE 2,3,4,5) show the comparison between the exact and numerical

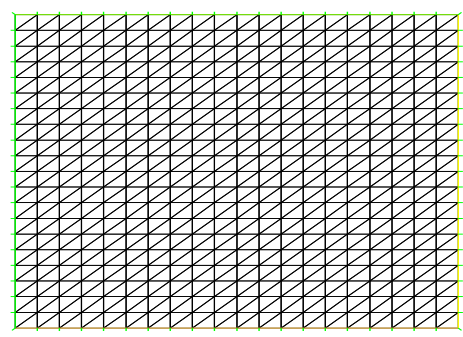

Figure 1. The initial mesh.

velocities for the times $t=0.3,0.5,0.7,1.24$. Furthermore, the figures (FIGURE 6,7 ) show the evolution of the corresponding meshes, which brings to light the power of the a posteriori error and corresponding bounds.
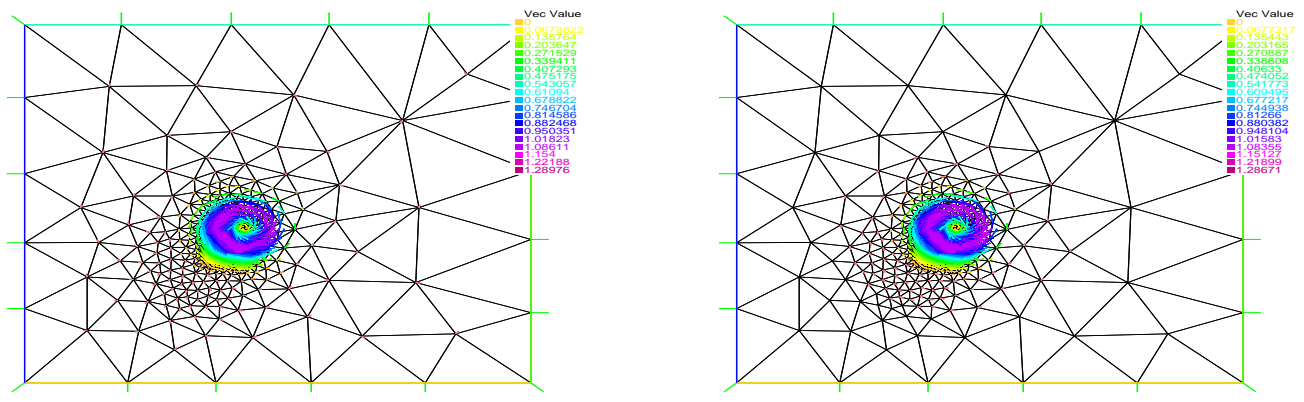

FiguRE 2. $\mathrm{t}=0.3$. Left: the numerical solution; right: the theoretical solution. 

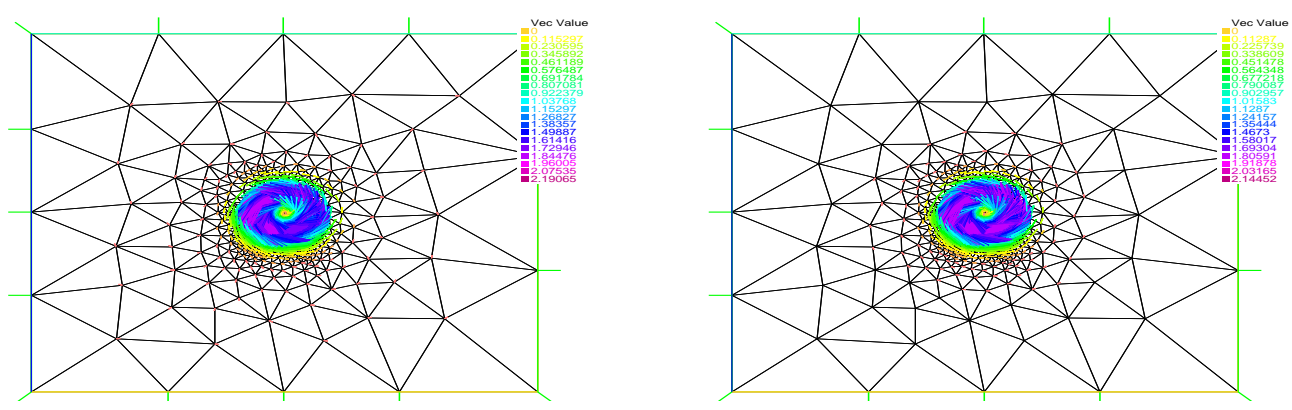

FIGURE 3. $\mathrm{t}=0.5$. Left: the numerical solution; right: the theoretical solution.
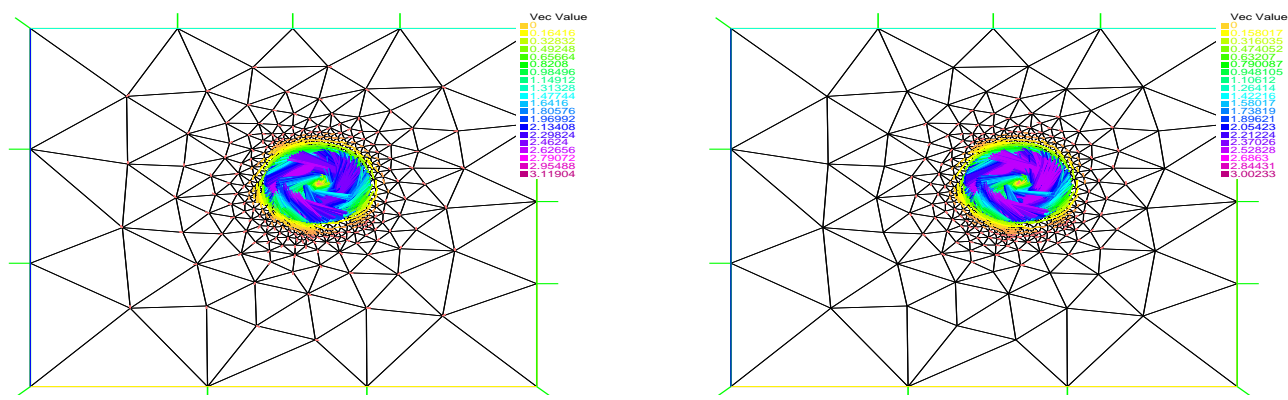

FIGURE 4. $\mathrm{t}=0.7$. Left: the numerical solution; right: the theoretical solution.
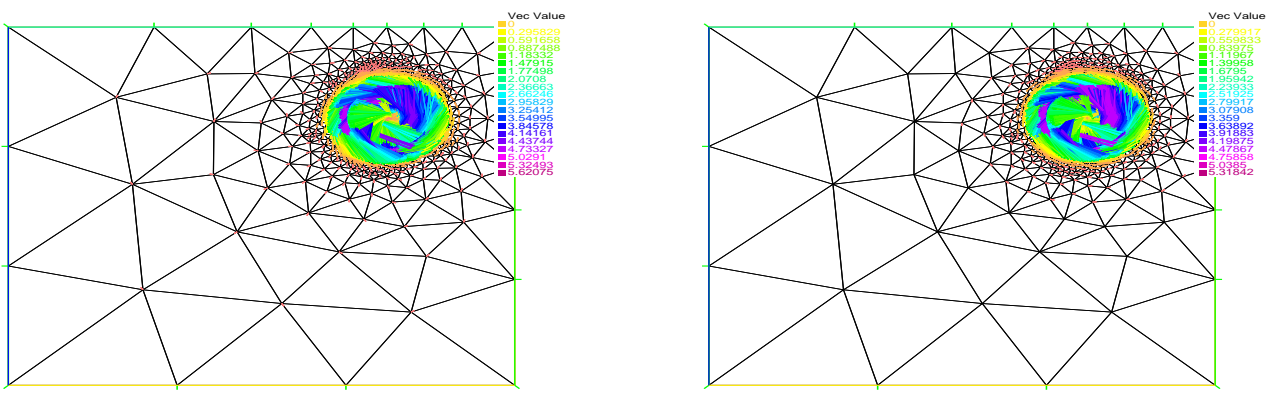

FiguRE 5. $\mathrm{t}=1.24$. Left: the numerical solution; right: the theoretical solution.

\section{REFERENCES}

[1] H. Abboud, F. El Chami \& T. Sayah, A priori and a posteriori estimates for three dimentional Stokes equations with non standard boundary conditions, Numer. Methods Partial Differential Equations, 28, pp. 1178-1193 (2012).

[2] C. Amrouche, C. Bernardi, M. Dauge \& V. Girault, Vector potentials in three-dimensional nonsmooth domains, Math. Meth. Applied Sciences, 2, pp. 823-864 (1998).

[3] A. Bergam, C. Bernardi, F. Hecht \& Z. Mghazli, Error indicators for the mortar finite element discretization of a parabolic problem, Numerical Algorithms, 34, pp. 187-201 (2003).

[4] C. Bernardi, F. Hecht \& R. Verfürth, Finite element discretization of the three-dimensional Navier-Stokes equations with mixed boundary conditions, Math. Model. and Numer. Anal., 3, pp 1185-1201 (2009).

[5] C. Bernardi, Y. Maday \& F. Rapetti, Discrétisations variationnelles de problèmes aux limites elliptiques, Collection "Mathématiques et Applications" 45, Springer-Verlag (2004).

[6] C. Bernardi \& T. Sayah, A posteriori error analysis of the time dependent Stokes equations with mixed boundary conditions, submitted for publication. 

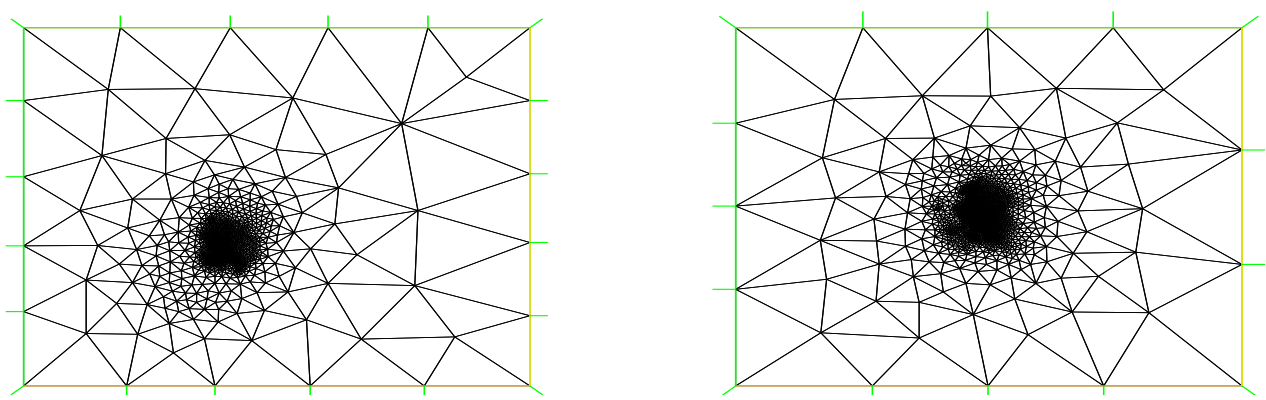

Figure 6. The mesh. Left : $\mathrm{t}=0.3 ;$ right $: \mathrm{t}=0.5$.
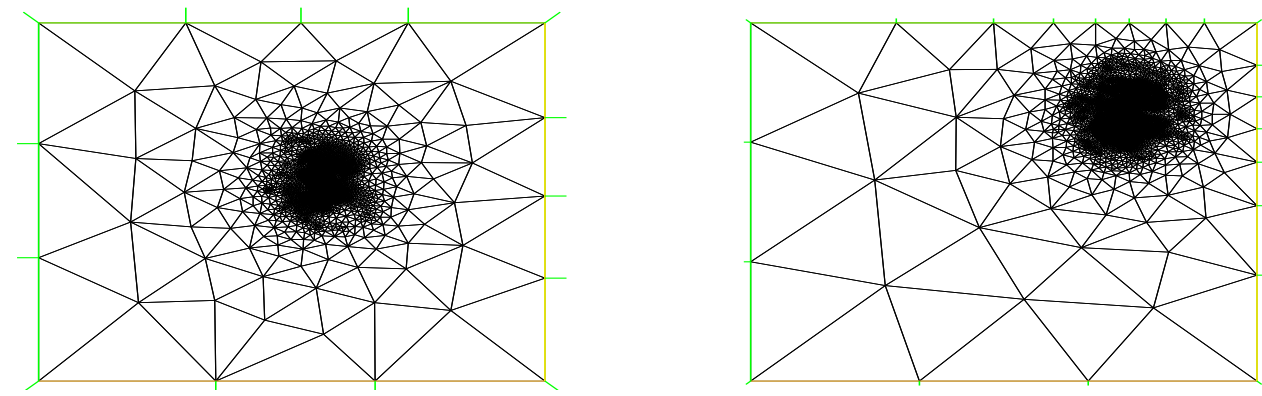

Figure 7. The mesh. Left $: \mathrm{t}=0.7 ;$ right $: \mathrm{t}=1.24$.

[7] C. Bernardi \& E. SüLI, Time and space adaptivity for the second-order wave equation, Math. Models and Methods in Applied Sciencesn 15, pp. 199-225 (2005).

[8] C. Bernardi \& R. Verfürth, A posteriori error analysis of the fully discretized time-dependent Stokes equations, Math. Model. and Numer. Anal., 38, pp. 437-455 (2004).

[9] P. Clément, Approximation by finite element functions using local regularisation, R.A.I.R.O. Anal. Numer., 9, pp.77-84 (1975).

[10] M. Costabel, A remark on the regularity of solutions of Maxwell's equations on Lipschitz domains, Math. Meth. in Appl. Sc. 12, pp. 365-368 (1990).

[11] F. El Chami \& T. Sayah, A posteriori error estimators for the fully discrete time dependent Stokes problem with some different boundary conditions, Calcolo, 47, pp. 169-192 (2010)

[12] V. Girault, P.-A. Raviart, Finite Element Approximation of the Navier-Stokes Equations, Lecture Notes in Mathematics 749, Springer-Verlag (1979).

[13] V. Girault \& P.-A. Raviart, Finite Element Methods for Navier-Stokes Equations, Theory and Algorithms, Springer, (1986).

[14] F. Hecht, New development in FreeFem++. J. Numer. Math., to appear (2013).

[15] J. Pousin \& J. Rappaz, Consistency, stability, a priori and a posteriori errors for Petrov-Galerkin methods applied to nonlinear problems, Numer. Math., 69, pp. 213-231 (1994).

[16] R. Temam, Navier-Stokes Equations. Theory and Numerical Analysis, Studies in Mathematics and its Applications, Vol. 2. North-Holland Publishing Co. (1977).

[17] R. Verfürth, A Review of A Posteriori Error Estimation and Adaptive Mesh-Refinement Techniques, Wiley and Teubner Mathematics (1996). 